\title{
I. Politische Arbeit unter den Kriegsgefangenen: Kooperation und Konkurrenz
}

An der politischen Arbeit unter den Kriegsgefangenen waren drei Instanzen beteiligt: die „Verwaltung des NKVD für die Angelegenheiten von Kriegsgefangenen und Internierten" (Upravlenie po delam voennoplennych i internirovannych UPVI), die Politische Hauptverwaltung der Roten Armee (Glavnoe političeskoe upravlenie raboče-krest’janskoj Krasnoj Armii - GlavPURKKA) und das Exekutivkomitee der Kommunistischen Internationale (EKKI). Diese versuchten ab dem Sommer 1941, deutsche Kriegsgefangene für eine ,antifaschistische Propaganda “ $z u$ nutzen. In den Kriegsgefangenenlagern erwuchs daraus eine sogenannte antifaschistische Bewegung (Antifa), deren Aktivisten seit dem Frühjahr 1942 auf eigens dafür eingerichteten Antifa-Schulen ausgebildet wurden. Mit Gründung des NKFD und sukzessive des Instituts 99 liefen alle diese Initiativen im Spätsommer 1943 in einer Koordinierungszentrale zusammen. Bis dahin aber konkurrierten UPVI, GlavPURKKA und EKKI um die Gestaltung der politischen Arbeit in den sowjetischen Kriegsgefangenenlagern.

\section{Verwaltung der Kriegsgefangenenlager}

Die „Verwaltung des NKVD für die Angelegenheiten von Kriegsgefangenen und Internierten" wurde im September 1939 gegründet. Sie war anfangs eine kleine und bescheiden ausgestattete Verwaltung, für die die Hauptverwaltung der Lager (Glavnoe upravlenie lagerej - GULag) aktiv Aufbauhilfe leisten mußte' ${ }^{1}$. Dessen ungeachtet arbeitete das UPVI relativ eigenständig. Anfang 1945 wurde es zu einer Hauptverwaltung aufgewertet ${ }^{2}$. Wie alle sowjetischen Lagerverwaltungen unterstand das UPVI dem Volkskommissariat für innere Angelegenheiten (Narodnyj kommissariat vnutrennych del - NKVD), wo es jedoch lange Zeit ein „Schattendasein “ führte 3 . Während der gesamten Dauer seines Bestehens (1939-1953) unterlag der Verwaltungsapparat der Kriegsgefangenenlager gewaltigen Schwankungen ${ }^{4}$. Ebenso veränderte sich die Zahl der Lager sehr stark. Von den acht beim deutschen

1 NKVD-Befehl vom 19. 9. 1939, in: Voennoplennye v SSSR, S. $72 \mathrm{f}$.

2 NKVD-Befehl vom 11.1. 1945, ebenda, S. $120 \mathrm{ff}$. Im weiteren wird durchgängig die Abkürzung UPVI und nicht das ab Januar 1945 innerhalb der sowjetischen Bürokratie gebräuchliche Kürzel GUPVI (Glavnoe upravlenie ...) verwendet.

3 Karner, Hauptverwaltung für Kriegsgefangene, S. 452.

41951 wurde die Hauptverwaltung (GUPVI) zurückgestuft zur Verwaltung (UPVI) und formal wieder dem GULag unterstellt. Die zentrale Leitung schrumpfte ab 1950 zusammen auf 39 Mitarbeiter; was ungefähr dem Mitarbeiterstand der Jahre 1939-41 entsprach; Karner, Archipel GUPVI, S. 78; siehe auch Hilger, Deutsche Kriegsgefangene, S. $82 \mathrm{ff}$. 
Überfall auf die Sowjetunion bestehenden Stammlagern existierten gegen Ende des Jahres 1941 nur noch drei, da die übrigen von der deutschen Wehrmacht überrannt worden waren. Im sowjetischen Hinterland mußten neue Lager aufgebaut werden. Im weiteren Verlauf des Krieges wuchs das Imperium des UPVI auf insgesamt 267 Lagerverwaltungen an, wobei jeder Lagerverwaltung nochmals mehrere Einzellager unterstanden ${ }^{5}$. Dabei befand sich während des Krieges ein großer Teil der Gefangenen in einem weitverzweigten Netz von Sammelpunkten und Aufnahmelagern, das vom UPVI nach dem sowjetischen Sieg bei Stalingrad Anfang Februar 1943 im Frontbereich angelegt wurde 6 . Diese Frontlager wanderten mit der Roten Armee nach Westen, wo sie nach der deutschen Kapitulation kurzfristig knapp 1,4 Millionen Gefangene aufnehmen mußten. Sie wurden bis zum Jahresende 1945 aufgelöst und die Kriegsgefangenen auf die Stammlager in der Sowjetunion verteilt ${ }^{7}$. Die Zahl der seit dem Juni 1941 aufgenommenen Kriegsgefangenen lag bis zum November 1942 bei knapp 20000. Durch den sowjetischen Sieg bei Stalingrad schnellte sie auf rund 170000 Kriegsgefangene hoch. Die großen Zuwächse für das UPVI kamen dann erst wieder ab dem Frühjahr 1944.

Für die politische Arbeit unter den Kriegsgefangenen fehlte der Verwaltung der Kriegsgefangenenlager sowohl das Personal als auch die organisatorische Struktur. Eine anfänglich geschaffene politische Abteilung war 1940 wieder aufgelassen worden, offenbar weil man sie nicht für notwendig erachtete ${ }^{8}$. Diese Einstellung änderte sich, als die Rote Armee in den Sommermonaten 1941 die ersten deutschen Kriegsgefangenen machte. Nun fuhren Offiziere der Politischen Hauptverwaltung der Roten Armee in die Lager, um die Möglichkeit zu erkunden, inwiefern deutsche Kriegsgefangene als Propagandisten gegen die Wehrmacht und das nationalsozialistische Regime verwendet werden könnten. Ein Politkommissar - Kirzanov - hielt sich vier Wochen in einem Lager bei Rjazan' auf, ein anderer Offizier - Bernikov fuhr in das Lager Javenga. Ihre anschließende Einschätzung war konträr. Kirzanov soll ein positives Fazit gezogen haben: Durch eine "gut organisierte Arbeit" könne ein Teil der Gefangenen von Hitler abgebracht werden ${ }^{9}$. Bernikov dagegen war sehr viel skeptischer: „Nach internen Berichten tadelten im allgemeinen nur drei von

5 Stand zum 1. 1. 1946; Übersicht in: Voennoplennye v SSSR, S. 1039; Karner (Im Archipel GUPVI, S. 246, Anm. 180) schätzt, daß es im Schnitt pro Lagerverwaltung zwölf Außenlager gab.

6 Im Frontbereich entwickelte sich mit vielen Umstrukturierungen bis zum Frühjahr 1944 ein gestaffeltes Lagersystem heraus: Aufnahmepunkte (PPV - priemnyj punkt voennoplennych) in 20-30 km Entfernung von der Front, Sammelpunkte (SPV - sbornyj punkt voennoplennych) in 50-70 km Entfernung und Durchgangslager (FPPL - frontovoj priemno-peresyl'nyj lager') in 100-150 km Entfernung; Galickij, Vražeskie voennoplennye v SSSR, S. 42 f.; vgl. Karner, Archipel GUPVI, S. 62; Übersicht in: Voennoplennye v SSSR, S. 1038; siehe auch Muchin, Das System der Gefangennahme, S. $114 \mathrm{ff}$.

7 In dieser Zeit entstanden unter der Verwaltung von 222 Lagerverwaltungen 2713 neue Einzellager. In der gleichen Zeit wurden allerdings 99 Lagerverwaltungen mit insgesamt 1080 Außenlagern aufgelöst; Rechenschaftsbericht des UPVI, RGVA/K 1p/23a/1, Bl. 31.

8 Karner, Hauptverwaltung für Kriegsgefangene, S. 452, Anm. 8; siehe den NKVD-Befehl vom 3. 12. 1940, in: Voennoplennye v SSSR, S. 82. Erst mit dem MVD-Befehl vom 19. 10. 1946 wurde wieder eine eigene Politabteilung des UPVI geschaffen, ebenda, S. 133.

9 Burzew, Einsichten, S. 63. 
Aufnabme von Kriegsgefangenen in der Zeit vom 22. Juni 1941 bis 26. Juni 1945 Zablenspiegel nach Berichten des UPVI ${ }^{10}$

bis zur Schlacht um Stalingrad:

$$
\begin{array}{llr}
22.06 .1941-31.12 .1941 & - & 9147 \\
01.01 .1942-18.11 .1942 & - & 10635
\end{array}
$$

bis zum sowjetischen Sieg bei Stalingrad:

19. $11.1942-31.12 .1942$

$01.01 .1943-03.02 .1943$

$\begin{array}{rr}- & 35747 \\ - & 115162 \\ & \\ - & 24919 \\ - & 40730 \\ - & 15351 \\ - & 520534 \\ - & 168970 \\ - & 789233\end{array}$

nach der deutschen Kapitulation:

08. 05. $1945-26.06 .1945$

$\begin{array}{r}-\quad 1390516 \\ \hline 3120944\end{array}$

hundert deutschen Kriegsgefangenen den Krieg gegen die Sowjetunion; aber sogar sie unterzogen dabei Hitler und sein System keiner Kritik. "11

Im Sommer 1941 verfügte aber auch die Politische Hauptverwaltung nicht über den für eine kontinuierliche Propagandaarbeit in den Kriegsgefangenenlagern notwendigen Apparat. Ihre 7. Verwaltung war nicht zuständig für die politische Beeinflussung von Kriegsgefangenen, sondern für die gegen den Feind gerichtete Frontpropaganda. Um allein dieser Aufgabe gerecht werden zu können, mußte sie nach dem deutschen Überfall auf die Sowjetunion aus Mangel an eigenen qualifizierten Propagandisten eiligst Philologen und sonstige Fachwissenschaftler aus sowjetischen Hochschulen rekrutieren. Auch wurden ausländische Kominternfunktionäre wegen ihrer Sprach- und Landeskenntnis gerne vom GlavPURKKA angeworben ${ }^{12}$. Somit war die 7. Verwaltung eine bunt zusammengewürfelte Abteilung, die noch im Aufbau befindlich war und für die Arbeit unter den Kriegsgefangenen kaum eine Hilfe sein konnte.

Die Grundlagen der politischen Arbeit unter den Kriegsgefangenen wurden hauptsächlich von Funktionären der Komintern gelegt, denn nur die Komintern konnte auf einen eingespielten Propagandaapparat zurückgreifen. Der Generalsekretär des Exekutivkomitees der Komintern (EKKI), Georgij Dimitrov, wurde so-

10 Registraturbericht des UPVI zum 1.3. 1944, Bericht für die Zeit vom 1.3. 1944 bis 1. 10. 1944, Berichte zum 20. 1. 1945 und 26. 6. 1945, RGVA/K 1p/01e, Akte 35, Bl. 45 und Bl. 72, ebenda Akte 46, Bl. 2 und ebenda Akte 39, Bl. 6; vgl. den Bericht des UPVI vom 27. 6. 1945, in: Voennoplennye v SSSR, S. 217; siehe auch Galickij, Vražeskie voennoplennye v SSSR, S. 39 ff.; siehe auch den statistischen Anhang von Hilger, Deutsche Kriegsgefangene, S. 390 ff.

11 Krupennikov, Die patriotische Lageeinschätzung von NKFD und BDO, S. 106.

12 Burzew, Einsichten, S. 31 ff.; Pike, Deutsche Schriftsteller im sowjetischen Exil 1933-1945, S. 489 ff.; Sebrow, Deutsche Schriftsteller im Kampf gegen den Faschismus, S. $160 \mathrm{ff}$. 
mit zur Koordinationszentrale. Dimitrovs entscheidende Rolle findet ihre Erklärung zudem darin, daß mit dem deutschen Überfall auf die Sowjetunion von der Komintern eine massive Rundfunkpropaganda gegen das nationalsozialistische Regime organisiert wurde. So kam es bereits am 22. Juni 1941 zu den ersten Absprachen zwischen EKKI und 7. Verwaltung des GlavPURKKA ${ }^{13}$. Am 6. Juli beschloß die sowjetische Führung: „Wir müssen die politische Arbeit unter den deutschen Kriegsgefangenen organisieren." 14

Unter erheblichen Anstrengungen wurde im August/September 1941 in Temnikov, dem damaligen zentralen Verteilungslager für deutsche Kriegsgefangene ${ }^{15}$, das erste „antifaschistische Lager-Aktiv“ aufgebaut. Neben der Entsendung der bereits erwähnten Politoffiziere der 7. Verwaltung fuhr im August 1941 eine Delegation der Komintern unter der Leitung von Walter Ulbricht nach Temnikov ${ }^{16}$. Die skeptische Einschätzung der 7. Verwaltung wurde dabei bestätigt. Obwohl man sich nur an Kriegsgefangene wandte, bei denen eine ablehnende Haltung gegenüber dem nationalsozialistischen Regime erwartet werden konnte - Überläufer und Söhne aus kommunistischen Arbeiterfamilien - , kam eine Gruppe von lediglich $30 \mathrm{Mann} z u-$ sammen, die schon bald nach Abreise der Delegation wieder auseinanderfiel. $\mathrm{Zu}$ groß war die Furcht der Kriegsgefangenen, nach dem als sicher angenommenen deutschen Sieg für die Zusammenarbeit mit dem Feind bestraft zu werden ${ }^{17}$. Nach der Abreise der Delegation blieben vier deutsche Exilkommunisten im Lager Temnikov. Während zwei von ihnen (Heinrich Dollwetzel und Oskar Stephan) in Zusammenarbeit mit der Lagerverwaltung die Besprechungen mit einzelnen Kriegsgefangenen weiterführten, gaben sich die anderen beiden (Otto Richter und Ferdinand Greiner) als Kriegsgefangene aus und lebten knapp einen Monat im Lager. Diese verdeckte Arbeit wurde im Jargon als „spezielle“ oder „besondere Arbeit" bezeichnet und sollte den Politfunktionären eine Einschätzung der Stimmungen unter den Kriegsgefangenen ermöglichen ${ }^{18}$. Sie wurde zum festen Bestandteil der

13 Selesnjow, Zur Hilfe Georgi Dimitroffs, S. 790 ff.; Burzew, Einsichten, S. 45; vgl. Dimitroff, Tagebücher, Einträge vom 22.6., 23.6., 28.6. und 1. 7. 1941.

14 Dimitroff, Tagebücher, Eintrag vom 6. 7.1941 zu einer Besprechung bei Molotov, der in den ersten Kriegstagen faktisch die Regierungsgeschäfte führte.

15 Ebenda, Eintrag vom 12. 9. 1941.

16 Die dazu notwendigen Absprachen traf Dimitrov mit Berija (NKVD) und Sudoplatov (NKGB), ebenda, Einträge vom 31.7. und 4. 8. 1941.

17 Krupennikov, Die politische Lageeinschätzung von NKFD und BDO, S. 106. Der Delegation gehörten an: Walter Ulbricht (KPD) und Jan Šverma (KPTsch) als Vertreter des EKKI, Kružkov, Kosoj und Draganov; „Bericht der Kommission über die Arbeit im Kriegsgefangenenlager von Temnikowski vom 4.-12. August 1941“, SAPMO-BArch RY 1/I 2/3, Bd. II,2 431, Bl. 4-15 (deutsche Fassung), RGASPI 495/10a/473, Bl. 1-13 (russische Fassung).

$18 \mathrm{Vgl}$. „Bericht über Fragen der deutschen, österreichischen und sudetendeutschen Kriegsgefangenen" vom 25.11. 1941, SAPMO-BArch RY 5/I 6/3/292, Bl. 1-33. In dem Bericht gibt es Rubriken über die soziale und altersmäßige Zusammensetzung der Kriegsgefangenen, „unzufriedene Stimmungen in der Armee“, Fragen nach der Wirkung der KPD-Rundfunk- und Flugblattpropaganda, die "Tätigkeit der Faschisten im Lager“, den „moralischen Zustand der Gefangenen“, „Fragen, über welche die Kriegsgefangenen diskutieren“ und „über die Lage in Deutschland" (Unzufriedenheit in der Bevölkerung, Lebensmittelversorgung, Stellung zum Krieg, Diskussionen über militärische Niederlagen). 
Antifa und führte zu dem oft beklagten Spitzelwesen in den Kriegsgefangenenlagern.

Im Lager Temnikov wurde ein sogenannter Politinstrukteur eingesetzt. Dieser Posten wurde - vermutlich ebenfalls im August 1941 - mit dem deutschen Exilkommunisten Heinrich Ewers besetzt. Ewers überredete und bestärkte einzelne Kriegsgefangene, öffentlich für die Beendigung des Krieges einzutreten. Die Formen eines solchen Engagements reichten von der Unterschrift unter einen entsprechenden Appell über regelmäßige Beiträge für die Lager-Wandzeitung, die Teilnahme an Gesprächskreisen im Lager bis hin zu Rundfunkappellen. Komintern und GlavPURKKA hatten nach ihren ersten Eindrücken schnell erkannt, daß ohne direkte Anleitung keinerlei Erfolge in der politischen Arbeit unter den Kriegsgefangenen zu erzielen sein würden. Aber auf die Kriegsgefangenen wurde kein Druck ausgeübt. Die ersten Aktivisten, die trotz aller Widerstände gefunden werden konnten, waren kooperationswillige Kommunisten, die eigens auf die Seite der Roten Armee übergelaufen waren, um Anschluß an die Exil-KPD zu bekommen. So waren von der Gruppe, die Ulbricht im August zusammengebracht hatte, zwei bis drei übergelaufene Wehrmachtsoldaten übriggeblieben, aus denen der Kern des AntifaAktivs geformt wurde ${ }^{19}$.

Als im Oktober 1941 abermals eine Delegation in das Lager Temnikov reiste ${ }^{20}$, konnte sie von einem bestehenden Antifa-Aktiv ausgehen. Bereits vor der Abreise erhielt daher die Kommission von Dimitrov die Vorgabe, ein „bedeutsames politisches Dokument" auszuarbeiten, „mit dem sich eine große Gruppe von gefangenen Soldaten an ihre Kameraden an der Front und im Hinterland wenden sollte, das den Bruch dieser Soldaten mit Hitler und dem faschistischen Regime und dem von ihm entfesselten Krieg demonstrieren sollte“21. Das Ergebnis dieser zweiten Delegationsreise war der von 158 Kriegsgefangenen unterschriebene „Appell an das deutsche Volk“, der in der ersten Ausgabe der aus diesem Anlaß neu gegründeten Kriegsgefangenenzeitung „Das Freie Wort“ veröffentlicht wurde22. Der „Appell der 158“ bildete bis zum Dezember 1942 die programmatische Basis der Antifa. Im Einklang mit den Aufrufen der Exil-KPD wurde in ihm mit der „Theorie von den zwei Deutschlands“, dem faschistischen und dem freiheitsliebenden, argumentiert: Hitler müsse gestürzt werden, damit ein freies, unabhängiges Deutschland Frieden mit der Sowjetunion schließen und das faschistische Regime in Deutschland überwunden werden könne. Zwar waren in dem Appell - wie auch in den später folgenden Aufrufen - die Zukunftsperspektiven vage gehalten, aber die Botschaft war eindeutig: „Hitlers Niederlage ist unvermeidlich! Hitlers Sturz ist des deutschen Volkes Rettung! " 23

19 Robel, Antifa, S. 34 f.; Wolff, Zur Beratung der 158, S. 46 f.; vgl. den Erinnerungsbericht von Heinz Ewers, in: Uhlig, Rückkehr aus der Sowjetunion, S. $183 \mathrm{f}$.

20 Neben den Kominternfunktionären Walter Ulbricht, Lotte Kühn, Paul Försterling und Jan Šverma reisten P.N. Fedossev (ZK der VKP (b)), K.L. Seleznev und Arthur Pieck (7. Verwaltung des GlavPURKKA) und P.N. Soprunenko (Chef des UPVI).

21 Selesnjow, Mit Walter Ulbricht im sowjetischen Kriegsgefangenenlager, S. 815; vgl. Dimitroff, Tagebücher, Eintrag vom 30. 9. 1941: „Beratung mit der neuen Delegation, die in das Kriegsgefangenenlager geschickt wird (...) Haben die Aufgaben der Delegation skizziert."

22 „Das Freie Wort“ erschien von November 1941 bis Juli 1943, siehe Selesnjow, Zur Geschichte der Zeitung „Das Freie Wort“, S. $951 \mathrm{ff}$.

23 "Appell an das deutsche Volk“, in: Sie kämpften für Deutschland, S. 114 ff.; Sywottek, Deut- 
Zur Evaluierung des in Temnikov verabschiedeten Appells fuhr Mitte Dezember 1941 erneut eine Delegation in ein Kriegsgefangenenlager. Dieses Mal wählte man das fern von Moskau gelegene Arbeitslager Spaso-Zavodsk in der Nähe von Karaganda ${ }^{24}$. Dort arbeitete als deutscher Politinstrukteur Heinrich Wielandt, der im November 1941 von Heinz Hoffmann und Erich Kundermann (beide KPD) Verstärkung erhalten hatte. Die im Dezember 1941 angereiste Delegation muß jedoch auf recht dürftige Anknüpfungspunkte gestoßen sein. Hoffmann urteilte über die Anfänge seiner Tätigkeit in Spaso-Zavodsk: „Natürlich war es unter diesen Bedingungen nicht möglich, eine systematische politische Schulungsarbeit zu betreiben, wie das später in den Antifaschulen geschah. Dazu hätte unsere Kraft nicht ausgereicht, und dazu bedurfte es auch eines Minimums an Bereitschaft unter den Kriegsgefangenen, über politische Fragen nachzudenken und zu diskutieren, sich mit der eigenen Vergangenheit auseinanderzusetzen und auch unter den harten Bedingungen der Kriegsgefangenschaft dem Leben einen Sinn zu geben. Deshalb bestand unsere erste Aufgabe darin, zu den Kriegsgefangenen, die zwar unsere Landsleute waren, uns aber als ihre Feinde betrachteten, Kontakt zu finden." 25 Ungeachtet dieser schwachen Grundlage war die Delegationsreise in das Lager Spaso-Zavodsk der Auftakt zur Intensivierung der Antifa.

Ab Januar 1942 delegierte die Komintern verstärkt ausländische Kommunisten als Politinstrukteure an das UPVI, damit sie beim Aufbau von Antifa-Aktivs in den Lagern halfen. Hatte sich bis dahin die politische Arbeit unter den Kriegsgefangenen auf die Lager Temnikov (Lager Nr. 58) und Spaso-Zavodsk (Lager Nr. 99) konzentriert, so kam im Frühjahr 1942 das Offizierslager Elabuga (Lager Nr. 97) hinzu ${ }^{26}$. Dort wurden der russische Instrukteur „Professor Arnold“27 und der deutsche Kommunist Otto Braun (Pseudonym „Wagner“) eingesetzt. Unter den Mannschaftsdienstgraden wirkten die deutschen Kommunisten Kurt Bürger, Georg Kassler und Arthur Lehmann als Politinstrukteure ${ }^{28}$. Die institutionelle Absicherung der von der Komintern delegierten Politinstrukteure erfolgte im August 1942 mit der Schaffung der „Gruppe Politinstrukteure“ als Unterabteilung des UPVI: „Die

sche Volksdemokratie, S. 116f.; vgl. Fischer, Sowjetische Deutschlandpolitik, S. 25 ff.; Robel, Antifa, S. $37 \mathrm{ff}$.

24 Der Kommission gehörten an: Soprunenko (UPVI), Seleznev, A. Pieck, Beljakov und Janzen (7. Verwaltung der GlavPURKKA), Lissovskij (ZK der VKP (b)) und die Kominternfunktionäre Walter Ulbricht, Lotte Kühn, Hans Mahle, Paul Försterling (KPD), Bela Szántó (KPU) und Bruno Köhler (KPTsch), Selesnjow, Reise mit deutschen Antifaschisten in ein Kriegsgefangenenlager, S. 278 ff.; vgl. Dimitroff, Tagebücher, Einträge vom 5. und 6.12. 1941; Kommissionsbericht in: Ulbricht, Zur Geschichte der deutschen Arbeiterbewegung, S. $266 \mathrm{ff}$.

25 Hoffmann, Moskau Berlin, S. 33; vgl. den Erinnerungsbericht von Hans Mahle, in: Uhlig, Rückkehr aus der Sowjetunion, S. $188 \mathrm{ff}$.

26 Vermutlich waren das alle Lager, in denen sich deutsche Kriegsgefangene befanden. Anfang 1942 verfügte das UPVI insgesamt nur über sieben Lager, siehe die Übersicht in: Voennoplennye v SSSR, S. 1028.

27 Das ist Abram Gural'skij, ein aus einer sowjetischen Hochschule von der 7. Verwaltung angeworbener Politinstrukteur. Scheurig (Verräter oder Patrioten, S. 42) bezeichnet ihn als "russischen Professor"; siehe auch Robel, Antifa, S. 45 und $49 \mathrm{ff}$.

28 Die Angaben beziehen sich auf den Stand vom Frühjahr 1942; Wolff, Die erste Konferenz, S. 277; vgl. den Erinnerungsbericht von Georg Kassler, in: Uhlig, Rückkehr aus der Sowjetunion, S. 187. 
Gruppe Politinstrukteure organisiert die antifaschistisch-propagandistische Arbeit unter den Kriegsgefangenen, Internierten und den Spezkontingenten ${ }^{29}$. Durch ihre Tätigkeit leitet sie die bei den Frontsammelpunkten und in den Lagern organisierten Klubs, roten Ecken [Wandzeitung], Bibliotheken und sonstige politisch-aufklärerische Einrichtungen. Sie versorgt die Lager mit politischer und schöngeistiger Literatur sowie Kulturgütern und organisiert die spezielle Arbeit unter den antifaschistischen Kriegsgefangenen. "30 Mit Hilfe der kommunistischen Emigranten in der Sowjetunion entstanden bis Januar 1943 Politabteilungen in 26 Lagerverwaltungen (von 40). Insgesamt zählte das UPVI 60 „Politinstrukteure mit Fremdsprachenkenntnissen" 31 , deren Zahl im Laufe des Jahres 1943 auf 108 anwuchs ${ }^{32}$. Alle als Instrukteure und Übersetzer delegierten Kominternfunktionäre wurden schließlich mit Beschluß des EKKI vom 5. Februar 1943 vollständig der Kaderverwaltung des NKVD bzw. des UPVI unterstellt ${ }^{33}$.

Dem UPVI gelang es nur mit der personellen Unterstützung der Komintern, seinen Politapparat bis zum Herbst 1942 aufzubauen. Das Frühjahr 1943 brachte dann gewaltige Umstellungen. Nach dem Sieg der Roten Armee bei Stalingrad Anfang Februar 1943 stieg die Zahl der Kriegsgefangenen sprunghaft an. Dadurch brach die Lagerverwaltung beinahe zusammen. Hatte man bis dahin versäumt, ein funktionierendes Netz an Aufnahmepunkten und Frontlagern zu schaffen, so mußten die erforderlichen organisatorischen Maßnahmen zu einem Zeitpunkt nachgeholt werden, zu dem die Rote Armee bereits über 200000 Gefangene im Frontbereich festhielt ${ }^{34}$. Allein auf dem Weg in die improvisiert angelegten Auffanglager um das vollkommen zerstörte Stalingrad herum kamen Zehntausende ums Leben ${ }^{35}$. Im Lager Beketovka bzw. seinen Außenlagern setzte ein unbeschreibliches Massensterben $e^{3}{ }^{36}$. Der Grund dafür lag nicht allein in den vollkommen unzureichenden Vorbe-

29 Spezialkontingente waren die aus deutscher Kriegsgefangenschaft befreiten Rotarmisten, die in sogenannten „Filtrierlagern" geheimdienstlich überprüft wurden. „Filtrierlager" entstanden auf der Grundlage eines NKVD-Befehls vom 27. 12. 1941. Zum Jahresbeginn 1943 gab es neun, ein Jahr später bereits vierzehn dieser Lager. Ihre Verwaltung wurde ab August 1944 von einer eigens dafür eingerichteten UPVI-Abteilung übernommen; Karner, Archipel GUPVI, S. 57; siehe auch Bonwetsch, Die sowjetischen Kriegsgefangenen, S. 141 f.; Semskow, Angst vor der Rückkehr, S. $161 \mathrm{f}$.

30 NKVD-Befehl Nr. 001603 vom 3. 8. 1942, RGVA/K 1p/37a/2, Bl. 26 RS-27; vgl. Karner, Archipel GUPVI, S. $57 \mathrm{f}$.

31 „Erfahrungen der politischen Arbeit unter den Kriegsgefangenen“, RGVA/K 1p/23a/8, Bl. 35 .

32 „Bericht über die Tätigkeit des UPVI in der Zeit des Großen Vaterländischen Krieges (Juni 1941 - März 1944)“, RGVA/K 1p/23a/2, Bl. 103. Die Zahl von 108 „Kader ohne militärischen Rang" bezieht sich auf Politinstrukteure aller in Moskau tätigen kommunistischen Parteien. Die KPD-Kaderabteilung zählte im September 1943 lediglich 16 KPD-Mitglieder; die als Übersetzer und 29, die als Politinstrukteure für das UPVI arbeiteten, SAPMO-BArch NY 4036/517, Bl. 43-44.

33 RGASPI 17/125/183, Bl. 3-4.

34 Das NKVD erließ den entsprechenden Befehl erst am 3. 2. 1943; Galickij, Tam v Beketovke, S. $19 \mathrm{f}$.; siehe Hilger, Deutsche Kriegsgefangene, S. $141 \mathrm{ff}$.

35 Overmans, Das andere Gesicht des Krieges, S. $431 \mathrm{ff}$;; Lehmann, Erinnerungen an die Kriegsgefangenschaft, S. 186.

$36 \mathrm{Zu}$ den nach wie vor schwierigen Zahlenberechnungen siehe Overmans (Das andere Gesicht des Krieges, S. 439 ff.) unter Auswertung der vorhandenen Literatur und Galickij (Tam v Be- 
reitungen des UPVI, sondern ebenso darin, daß die deutschen Soldaten bereits ausgehungert, unzulänglich gekleidet und viele von ihnen mit schweren Erfrierungen in Gefangenschaft gingen ${ }^{37}$. Der Einsatz mehrerer sowjetischer Kommissionen zeigt aber, daß wegen der Zustände in Beketovka Klärungsbedarf herrschte. UPVI und Rote Armee schoben sich gegenseitig die Verantwortung zu. In dem NKVDBericht an Stalin wurde betont, daß allein 33111 Kriegsgefangene gestorben seien, bevor sie überhaupt die Aufnahmepunkte des UPVI erreicht hätten, und das UPVI folglich dafür keine Verantwortung trüge. Zugleich gibt dieser Bericht einen unfreiwilligen Einblick in die Schwierigkeiten des UPVI: 221261 Kriegsgefangene befänden sich noch im Frontbereich - in dem die UPVI keinerlei Vorbereitungen getroffen hatte - und lediglich 35657 Kriegsgefangene befänden sich in den Lagern im Hinterland ${ }^{38}$.

Infolge der chaotischen Zustände in den Frontlagern wurde im Mai 1943 der bisherige UPVI-Chef, Major Petr Soprunenko, durch Generalmajor Ivan Petrov ersetzt. Das gesamte UPVI wurde reorganisiert und offensichtlich, dafür spricht der wesentlich höhere Dienstgrad Petrovs sowie die personelle Aufstockung des Verwaltungsapparates, in seiner Bedeutung aufgewertet ${ }^{39}$. Dem neuen Chef wurden insgesamt zehn Stellvertreter zur Seite gegeben ${ }^{40}$. Fünf von ihnen waren verantwortlich für bestimmte Aufgabenbereiche innerhalb der Leitung auf zentraler Ebene, die anderen fünf für das sich ausbreitende Netz von Lagern im Frontbereich und im Hinterland ${ }^{41}$. Der Leiter des UPVI unterstand nun direkt dem Volkskommissar für Innere Angelegenheiten, Lavrentij Berija bzw. dessen für die Belange des UPVI verantwortlichen Stellvertreter Sergej Kruglov. Die Zusammenarbeit des UPVI mit anderen Volkskommissariaten - Vereinbarungen über die Aufnahme, den Transport, die Ernährung und die medizinische Versorgung der Kriegsgefangenen wurde durch das UPVI realisiert, das dabei jedoch immer auf Anweisung des NKVD handelte.

Etwas komplizierter gestalteten sich die Zuständigkeiten bei der im Mai 1943 neu

ketovke, S. $18 \mathrm{ff}$.) unter Auswertung von Dokumenten aus dem RGVA/K, allerdings ohne den Versuch einer Gesamtwertung.

37 Overmans, Das andere Gesicht des Krieges, S. 419.

38 Berija an Stalin am 26. 2. 1943, RGVA/K 1p/9a/8, Bl. 41. Die Zahl von rund $221000 \mathrm{im}$ Frontbereich befindlichen Kriegsgefangenen bezieht die aus deutscher Hand befreiten Rotarmisten und Hilfswilligen mit ein. Schätzungen (Overmans, Das andere Gesicht des Krieges, S. 441) gehen von rund 77000 Hilfswilligen und ca. 5000 sowjetischen Kriegsgefangenen aus; siehe auch Gorbunov, Deutsche Kriegsgefangene in der Sowjetunion, S. 46 und S. $92 \mathrm{f}$.

39 Karner, Archipel GUPVI, S. 61 f.; vgl. NKVD-Befehl vom 24. 2. 1943, in: Voennoplennye v SSSR, S. $99 \mathrm{f}$.

40 In der sowjetischen Verwaltung ist es üblich, daß ein Behördenchef mehrere Stellvertreter hat, wobei jeder für bestimmte Bereiche verantwortlich ist. Die Stellvertreter entsprechen den Abteilungsleitern einer deutschen Behörde.

41 Vgl. "Struktur des UPVI und Verteilung der Aufgaben“ vom 10.5. 1943, RGVA/K $1 \mathrm{p} / 9 \mathrm{a} / 13, \mathrm{Bl} .1-3$ und „Verteilung der Verantwortungsbereiche unter den Stellvertretern des Chefs des UPVI" vom 24. 6. 1943, RGVA/K 1p/23a/2, Bl. 3-9; vgl. dic Wiedergabe bei Karner (Hauptverwaltung für Kriegsgefangene und Internierte, S. 469f.), bei der jedoch ein in diesem Zusammenhang wichtiger Zusatz in dem Dokument fehlt: Die operative Abteilung, ursprünglich Ratušnyj unterstellt, wurde mit einem handschriftlich eingefügten Pfeil Mel'nikov zugeordnet. Die Zuständigkeit Mel'nikovs ergibt sich - wie im weiteren noch zu sehen sein wird - zweifelsfrei aus der in den Lagern durchgeführten operativen Arbeit. 
geschaffenen „operativ-čekistischen Abteilung“ des UPVI. Die Aufgabe dieser Abteilung war - in bezug auf die Kriegsgefangenen - die Überwachung der Lagerinsassen (z.B. Aufdeckung von Fluchtvorbereitungen), die Durchführung von Ermittlungen (z.B. gegen Kriegsverbrecher) und die Beschaffung von Informationen durch Befragungen bzw. Verhöre, die auch das Abschöpfen von Informationen durch Spitzel und Abhöranlagen einschloß ${ }^{42}$. Diese Aufgaben waren bislang von der operativen Gruppe des NKVD unter Leitung von Ivan Serov übernommen worden. Serov, einer der Stellvertreter Berijas, war im Volkskommissariat des Inneren ebenso für das UPVI zuständig wie der ebenfalls stellvertretende NKVD-Chef Kruglov. Diese Doppelbesetzung ergab sich aus dem Umstand, daß das NKVD im Juli 1941 mit dem Volkskommissariat für Staatssicherheit (NKGB -Narodnyj komissariat gosudarstvennoj bezopasnosti) zusammengelegt worden war. $\mathrm{Zu}$ einer Entflechtung der Sicherheitsapparate kam es erst im April 1943. Die Staatssicherheit wurde ein selbständiges Volkskommissariat und der militärische Geheimdienst (SMERŠ - smert' špionam) aus dem NKVD ausgegliedert und dem Volkskommissariat für Verteidigung (NKO - Narodnyj kommissariat oborony) unterstellt ${ }^{43}$. Nun erhielt auch das UPVI einen eigenen Sicherheitsapparat. Dieser wurde geleitet vom ersten Stellvertreter des UPVI-Chefs, General Nikolaj Mel'nikov, der hinsichtlich der „speziellen Fragen der Arbeit“ mit SMERŠ kooperierte ${ }^{44}$. Im Frühsommer 1944 übernahm Oberst Švec - möglicherweise kommissarisch - die Leitung der operativen Abteilung, die ab Anfang 1945 von General Amajak Kobulov geleitet wurde ${ }^{45}$. Die operative Abteilung übernahm alle „Sonderaufgaben“ innerhalb des UPVI, d.h. geheimdienstlich oder politisch brisante Aufgaben. So waren die vom UPVI im Zusammenhang mit der Gründung des Nationalkomitees „Freies Deutschland“ eingerichteten Sonderlager „operative Objekte“ und unterstanden der direkten Kontrolle Mel'nikovs. Während das „Objekt Nr. 15 “ in Lunevo das Lager für die NKFD-Mitglieder war, waren die anderen „Objekte“ (Nr. 20 in Planernoe, Nr. 25 in Dombrovo, Nr. 35 in Ozery) Datschen im Moskauer Umland. Dort wurden prominente Kriegsgefangene „operativ bearbeitet“" d.h. eingehenden Gesprächen mit Politoffizieren unterzogen, gegebenenfalls verhört oder über $\mathrm{Ab}$ höranlagen ausgehorcht ${ }^{46}$.

Im Mai 1943 wurde außerdem eine Unterabteilung des UPVI für die politische Arbeit unter den Kriegsgefangenen gegründet. Verantwortlicher Abteilungsleiter war Petrovs „Stellvertreter für die politische Abteilung“, Oberst Jakovec. Auf der zentralen Verwaltungsebene war das UPVI weiterhin ausdrücklich zur Zusammen-

42 Siehe die umfangreichen Abhörberichte, die das UPVI bzw. das NKVD an Stalin sandte, in: Archiv novejšej istorii Rossii, tom I; Kozlov, Politische Einstellung und Stimmung, S. $113 \mathrm{ff}$.

43 Lubjanka, S. 34.

44 „Struktur des UPVI und Verteilung der Aufgaben" vom 10.5. 1943, RGVA/K 1p/9a/13, Bl. 1-2.

45 Am 7. 4. 1944 verübte Mel'nikov „aus persönlichen Motiven“ Selbstmord, Reschin, General zwischen den Fronten, S. 169. Leider bleibt Reschin den Beleg schuldig, siehe auch Voennoplennye v SSSR, S. 1078. Auch aus den Quellen geht die - im Laufe des Jahres 1944 offensichtlich leitende - Tätigkeit von Šv ec eindeutig hervor, während Mel'nikov ab dieser Zeit nicht mehr in Erscheinung tritt.

46 „Plan über die Arbeit mit den kriegsgefangenen deutschen Generalen“, in: Rešin, Sojuz nemeckich oficerov, S. 102. Im Objekt Nr. 35 (Ozery) wurde Generalfeldmarschall Paulus interniert. 
arbeit mit der Politischen Hauptverwaltung der Roten Armee verpflichtet ${ }^{47}$, aber in den einzelnen Lagerverwaltungen war einzig und allein Jakovec für die Durchführung der politischen Arbeit zuständig. Ihm unterstanden die Politinstrukteure, die von der Komintern an das UPVI überstellt worden waren ${ }^{48}$.

Insgesamt gestaltete sich die politische Arbeit in den Kriegsgefangenenlagern nach wie vor schwierig. Sie war ein Zusammenspiel von politischer und operativer Abteilung des UPVI, GlavPURKKA, Geheimdiensten und Komintern. Die in der im Mai 1943 festgelegten Aufgabenverteilung des UPVI genannten „speziellen Bestimmungen" für die inhaltlichen Ausarbeitungen der politischen Abteilung lassen zudem erahnen, daß im Gegensatz zu den klar umrissenen Verwaltungsaufgaben des UPVI die politischen Entscheidungen zusammen mit anderen Instanzen getroffen werden mußten. Dabei spielte die Politische Hauptverwaltung der Roten Armee eine maßgebliche Rolle.

\section{Politische Hauptverwaltung der Roten Armee}

Die 7. Verwaltung der Politischen Hauptverwaltung der Roten Armee war sehr an an der politischen Arbeit mit den Kriegsgefangenen interessiert. Man wollte sie für die Frontpropaganda nutzen. Zu direkten Propagandaeinsätzen von Kriegsgefangenen an der Front durch die 7. Verwaltung kam es erst ab Anfang 1943 (Stalingrad) und später vor allem im Zusammenhang mit dem Nationalkomitee „Freies Deutschland" 49 , aber die 7. Verwaltung versuchte von Anfang an, sich einen institutionellen Zugriff auf Kriegsgefangene zu sichern. Bereits im Juni 1941 schuf sie die Unterabteilung "Arbeit mit den Kriegsgefangenen“, und im Zusammenhang mit dem Ausbau der Antifa entstanden später weitere Unterabteilungen ${ }^{50}$. Auch wenn die 7. Verwaltung damit institutionell über diejenigen Abteilungen verfügte, die das UPVI bis zum Frühjahr 1943 nicht hatte, so fehlten ihr ebenso die qualifizierten Mitarbeiter, um die politische Arbeit durchführen zu können. Aber sie besaß die besseren Voraussetzungen, um ihren Führungsanspruch durchsetzen zu können.

Die 7. Verwaltung war zwar eine Abteilung der Politischen Hauptverwaltung, sie war politisch aber unmittelbar an die Weisungen des „Sowjetischen Büros für militärpolitische Propaganda“" (Sovetskoe bjuro voenno-političeskoj propagandy) gebunden, das am 25. Juni 1941 als Koordinierungsstelle zwischen Roter Armee und VKP (b) geschaffen worden war. Es sollte die Inhalte der Propaganda unter den Truppen des Gegners festlegen, entsprechende Flugblätter und Losungen erarbeiten und deren Verbreitung an der Front organisieren. Die 7. Verwaltung galt als "Arbeitsorgan“ des Büros und war damit zwei Herren verpflichtet ${ }^{51}$. Diese Art von Doppelzuständigkeiten war ein in der sowjetischen Bürokratie häufig anzutreffen-

47 „Struktur des UPVI und Verteilung der Aufgaben“ vom 10.5. 1943, RGVA/K 1p/9a/13, Bl. 1.

48 Ebenda, Bl. 2.

49 Dazu siehe Burzew, Deutsche Antifaschisten, S. 421; ders., Učastie germanskich antifašistov, S. $41 \mathrm{ff}$.

50 Burzew, Einsichten, S. 92.

51 Rechenschaftsbericht des Büros für militärpolitische Propaganda vom April 1942, RGASPI 495/77/17, Bl. 41; vgl. Burzew, Einsichten, S. 36 f. 
des Phänomen. Es wurde verstärkt durch die Schaffung ressortübergreifender Koordinierungsgremien, die insbesondere für die Mobilisierung der sowjetischen Staats- und Wirtschaftsverwaltung nach dem deutschen Überfall im Sommer 1941 eine wichtige Rolle spielten ${ }^{52}$. Den Vorsitz im Büro für militärpolitische Propaganda führte nominell der Chef der Politischen Hauptverwaltung Lev Mechlis. Der entscheidende Mann war jedoch Aleksandr Ščerbakov, der schließlich im Juni 1942 offiziell die Leitung der Politischen Hauptverwaltung übernahm. Das Büro für militärpolitische Propaganda wurde umgewandelt in den „Rat für militärpolitische Propaganda" (Sovet voenno-političeskoj propagandy) und dem GlavPURKKA unterstellt. Der Rat wurde somit zum Regelorgan innerhalb der Politischen Hauptverwaltung und seine Mitglieder mit Ausnahme von Dimitrij Manuil'skij und Mechlis alle neu benannt. Vorsitzender des Rates war nun Ščerbakov ${ }^{53}$.

An der Rolle Ščerbakovs wird deutlich, daß die schwer durchschaubaren und verwirrenden Zuständigkeiten innerhalb der sowjetischen Bürokratie klarer zuzuordnen sind, wenn man sich nicht auf die Institutionen, sondern auf die personellen Verflechtungen - Umbesetzungen, Gremienzusammensetzungen, Kompetenzzuweisungen - konzentriert ${ }^{54}$. Ščerbakov war Kandidat des Politbüros und hatte als Leiter des Sowjetischen Informationsbüros (Sovinform - Sovetskoe informacionnoe bjuro) die Kontrolle über die gesamte Presse, den Rundfunk und die zentralisierte Kriegsberichterstattung. Auch die „Organisation der Gegenpropaganda gegen die deutsche und sonstige feindliche Propaganda" galt als Aufgabe des Sovinform ${ }^{55}$. Hinsichtlich seiner mächtigen Stellung erstaunt es zwar, daß er nicht Mitglied des Politbüros und des Staatlichen Verteidigungskomitees - dem „Kriegskabinett" - war ${ }^{56}$, aber Ščerbakov war zweifelsohne der für die politische Arbeit unter den Kriegsgefangenen entscheidende Spitzenfunktionär. Von Anbeginn an ließ er sich unmittelbar über alle Schritte der politischen Arbeit in den Kriegsgefangenenlagern berichten ${ }^{57}$. Er war „letztlich jene Instanz, in der die endgültigen Entschei-

52 „Diese Sonderorgane zeichneten sich im wesentlichen dadurch aus, daß sie eine funktional, oft auch regional gelegentlich zeitlich umrissene Aufgabe zu erfüllen hatten, daß sie außerordentliche Vollmachten besaßen, daß sie Regelinstanzen beaufsichtigten, korrigierten oder überspielten, und daß sie häufig von einer Person aus dem innersten Machtkreis geleitet wurden oder einer solchen Person unterstellt waren." Segbers, Die Sowjetunion im Zweiten Weltkrieg, S. 48.

53 Hervorzuheben ist die Ratsmitgliedschaft des Politbüromitglieds Andrej Ždanov, die auf eine politische Aufwertung des Rates schließen läßt. Der Rat wurde auf Beschluß des ZK der VKP (b) vom 12. 6. 1942 gegründet. Neue Mitglieder des Rates waren außer den bereits genannten: E.M. Jaroslavskij, I.V. Rogov (Chef der Politischen Hauptverwaltung der Kriegsflotte), G.F. Aleksandrov (Leiter der Propagandaabteilung des ZK der VKP (b)) und F.F. Kuznecov (stellvertretender Chef des GlavPURKKA), Sablin, O dejatel'nosti soveta voenno-političeskoj propagandy, S. 91; Sovetskaja Voennaja Ėnciklopedija, tom 7, S. 407.

54 Segbers, Die Sowjetunion im Zweiten Weltkrieg, S. $66 \mathrm{ff}$.

55 Dem Sovinform gehörten an: Ščerbakov (Leiter), Lozovskij, Chavinson, Polikarpov, Saksin und Golikov; „Beschluß des ZK der VKP (b) und des SNK der UdSSR über die Gründung und die Aufgaben des Sowjetischen Informationsbüros" vom 24. 7. 1941, in: KPSS v rezoljucijach i rešenijach, tom 7, S. 213.

56 Dennoch zählt Segbers (Die Sowjetunion im Zweiten Weltkrieg, S. 51) ihn zu der 29-köpfigen Machtelite der Sowjetunion während des Krieges.

57 So ging der erste Bericht über die Stimmung der Kriegsgefangenen im Lager Temnikov vom August 1941 direkt an Ščerbakov, Ulbricht an Ščerbakov am 18. 8. 1941, SAPMO-BArch RY 1/I 2/3, Bd. II,2/431, Bl. 53; vgl. Ulbricht an Dimitrov am 21.8. 1941: „Soweit ich erfahren 
dungen über Angelegenheiten hinsichtlich der Kriegsgefangenen getroffen wurden, sei es zur Zeit der Komintern oder später des NKFD“58.

Im Frühsommer 1942 wurden die Führungsstrukturen in der gesamten Informations- und Propagandapolitik der Sowjetunion gestrafft und in der Person Ščerbakovs unter eine einheitliche politische Leitung gestellt ${ }^{59}$. Die laufende Arbeit sowohl des Büros als auch des Rates für militärpolitische Propaganda lag dabei in der Hand Manuil'skijs, der als Sonderbeauftragter Ščerbakovs bezeichnet werden kann. Zwar besaß Manuil'skij innerhalb der sowjetischen Machtelite keine grundlegende politische Entscheidungskompetenz, aber auf dem Wege von Vorschlägen und durch die Ausführung meist allgemein gehaltener politischer Vorgaben hatte er einen nicht unbeträchtlichen Handlungsspielraum ${ }^{60}$. Er war seit Juni 1941 Mitglied des Büros für militärpolitische Propaganda und wurde im Juli 1942 offiziell zum GlavPURKKA abkommandiert ${ }^{61}$. Manuil'skijs Einfluß auf die politische Arbeit unter den Kriegsgefangenen war verquickt mit der propagandistischen Tätigkeit der Komintern, deren Führungsspitze er zugleich angehörte. Die Umstrukturierung des Büros für militärpolitische Propaganda beeinträchtigte seine Tätigkeit genauso wenig wie die im Mai 1943 folgende Auflösung der Komintern. Erst als im Juli 1944 der Rat für militärpolitische Propaganda aufgelöst wurde, verließ er die 7. Verwaltung und trat in der politischen Arbeit unter den Kriegsgefangenen nicht mehr in Erscheinung62.

Manuil'skij war der geistige Vater des Nationalkomitees „Freies Deutschland“, dessen Idee er erstmals im Frühjahr 1942, möglicherweise im Zuge der sich anbahnenden Veränderungen in der sowjetischen Informations- und Propagandapolitik, vorbrachte. Zusammen mit Solomon Lozovskij - stellvertretender Leiter des Sovin-

konnte, beschäftigt sich im ZK Genosse Scherbakow mit diesen Fragen [der politischen Arbeit unter den Kriegsgefangenen]", RGASPI 495/77/30, Bl. 3.

58 Babičenko, Zur Neubewertung der Zusammenarbeit, S. 81.

59 Burzew (Einsichten, S. 9), der damalige Leiter der 7. Verwaltung, spricht im Zusammenhang mit der Schaffung des Rates für militärpolitische Propaganda von der „vollständigen Integration der Propagandaarbeit in die Arbeit der Partei“.

60 Babičenko, Zur Neubewertung der Zusammenarbeit, S. 82: „Er war faktisch nach Ščerbakov die zentrale Person, die sich in der Parteiführung mit den Fragen der Gefangenen beschäftigte, wie übrigens auch im Exekutivkomitee der Komintern nach Dimitrov. Oft war Manuil'skij nicht nur Kontrolleur, sondern auch Verfasser von politisch wichtigen, die Arbeit unter den Kriegsgefangenen betreffenden Dokumenten. “; vgl. Segbers, Die Sowjetunion im Zweiten Weltkrieg, S. 71: „Ein die Zentralisierung mit der Flexibilisierung verbindendes Prinzip war die gezielte Bindung von außerordentlichen Kompetenzen an Personen. Auch hier ist nicht nur an die Spitze zu denken, wo sich Macht, Aufträge und Zuständigkeiten bei wenigen Personen häuften."

61 Burzew, Einsichten, S. 101.

62 Ebenda, S. 271; Manuil'skij, ein gebürtiger Ukrainer, wurde im Juli 1944 ukrainischer Volkskommissar für Auswärtige Angelegenheiten und später Vertreter der Ukraine in den Vereinten Nationen. Manuil'skij war insgesamt einflußreicher, als seine Amtsfunktionen vermuten lassen, Sadekova, Dimitri Manouilski, S. 53; vgl. die zahlreichen Einträge in Dimitrovs Tagebuch. So gehörte Manuil'skij ab dem 23.6. 1941 zur „ständigen Leitung“ des EKKI, nahm am 6. 7. 1941 an dem Spitzengespräch bei Molotov zur Organisation der politischen Arbeit unter den Kriegsgefangenen teil und kam immer wieder zu den Koordinierungstreffen bei Dimitrov hinzu (1.11., 10.11., 9. 12. 1941). Am 7. 4. 1942 schließlich besprach Dimitrov mit ihm „die Aufteilung seiner Arbeit (er soll zu $80 \%$ in der Politverwaltung der Roten Armee arbeiten)". 
form und als solcher Stellvertreter Ščerbakovs - verfaßte er im April 1942 ein Papier, in dem er die Bildung eines „Anti-Hitler-Komitees“ aus deutschen Emigranten in der Sowjetunion und Kriegsgefangenen vorschlug. Hintergrund war, daß Ende 1941 in Absprache mit Ščerbakov begonnen worden war, alle Gruppen der sowjetischen Gesellschaft im Kampf gegen den Faschismus in antifaschistischen Komitees zu organisieren. Bereits im August 1941 war unter Lozovskijs unmittelbarer Anleitung mit der Schaffung eines Allslawischen Komitees begonnen worden. Kurz darauf folgte die Gründung eines jüdischen antifaschistischen Komitees ${ }^{63}$. Nach Lozovskijs Aussagen waren die antifaschistischen Komitees bewußt überparteilich und allein für den gemeinsamen Kampf gegen den Faschismus und die Unterstützung der Sowjetunion gedacht gewesen. Alle organisatorischen Schritte zur Schaffung der Komitees seien mit Ščerbakov bzw. dem ZK der VKP (b) abgesprochen gewesen ${ }^{64}$. Waren schon zuvor deutsche Schriftsteller (Johannes R. Becher, Friedrich Wolf) an den sogenannten Meetings beteiligt gewesen, so versuchten Lozovskij und Manuil'skij im Frühjahr 1942 offenbar, diese Ansätze zu einem eigenständigen Komitee deutscher Antifaschisten auszubauen. Der Vorschlag zur Gründung eines deutschen antifaschistischen Komitees wies zumindest unübersehbare Analogien auf, zumal die beiden Autoren in unmittelbarer Verbindung zu Ščerbakov standen. Hinzu kam der Tagesbefehl Stalins zum Jahrestag der Roten Armee am 23. Februar 1942, den Manuil'skij und Lozovskij zum Anlaß ihrer Initiative nahmen. Stalin betonte darin die Schwere des Verteidigungskrieges, der die Rote Armee zu einem harten Kampf gegen die Wehrmacht zwinge. Um aber die letztlich friedlichen Absichten der Sowjetunion zu bekräftigen, prägte er den später viel zitierten Ausspruch, „daß die Hitler kommen und gehen, aber das deutsche Volk, der deutsche Staat bleibt" 65 .

Manuil'skij und Lozovskij griffen die Argumentation Stalins auf und kombinierten sie mit der Idee antifaschistischer Komitees. In ihrem Brief vom 4. April 1942 an Stalin und Molotov verwiesen sie darauf, daß „nicht alle deutsche Soldaten, die den Befehl Stalins gelesen haben, glauben, daß sie nicht erschossen werden, wenn sie sich gefangen geben“. Mit offizieller sowjetischer Unterstützung solle daher ein „Komitee aus Persönlichkeiten des öffentlichen Lebens Deutschlands zur Erleichterung der Gefangengabe deutscher Soldaten“ gegründet werden, das sich zusammensetzt aus „allen Deutschen, die den bekannten Aufruf an das deutsche Volk unterschrieben haben"66. In diesem Aufruf vom 25. Januar 1942 hatten 60 in die Sowjetunion emigrierte KPD-Mitglieder zur Schaffung von Soldatenkomitees und zur Gehorsamsverweigerung in der Wehrmacht aufgerufen: „Schafft in allen Truppenteilen und Einheiten Soldatenkomitees für den Kampf gegen den Krieg und gegen

63 Petrova, Antifašistskie komitety v SSSR, S. $70 \mathrm{ff}$.

64 Nepravednyj sud, S. 147.

65 Befehl des Volkskommissars für Verteidigung Nr. 55 vom 23. 2. 1942, in: Stalin, Über den Großen Vaterländischen Krieg, S. 31 ff.; Meissner (Rußland, die Westmächte und Deutschland, S. 13) sieht in dem Stalin-Befehl eine Variante der sowjetischen Politik, „mit jenen nationalistischen Kräften ins Gespräch zu kommen, die einen Sturz des nationalsozialistischen Regimes am ehesten beschleunigen konnten und zugleich einer Zusammenarbeit selbst mit einem bolschewistsichen Rußland nicht abgeneigt waren".

66 Manuil'skij und Lozovskij an Stalin und Molotov am 4. 4. 1942 „über die Verstärkung der Arbeit zur Zersetzung der deutschen Armee“, RGASPI 495/77/30, Bl. 19-20. 
Hitler! Kehrt eure Waffen gegen den Unmenschen und Blutsäufer Hitler, gegen das verbrecherische Naziregime! Geht mit euren Waffen zur Roten Armee über!"67 In einem internen Papier vom 3. April 1942 - wahrscheinlich die Tischvorlage für die Beratung des Büros für militärpolitische Propaganda - hatten Manuil'skij und Lozovskij ihre Idee näher ausgeführt. Das Komitee solle für den freiwilligen Rückzug der Wehrmacht eintreten bzw. eingekesselte Wehrmachtsverbände dazu auffordern, mit der Roten Armee über eine kollektive Gefangengabe zu verhandeln. Neben der Entsendung von Bevollmächtigten an die Front dachten Manuil'skij und Lozovskij auch an den Einsatz von Kampf- und Propagandabrigaden im deutschen Hinterland, aber dieser Gedanke wurde fallen gelassen. In der Argumentation der Autoren diente die Gründung des Komitees in allererster Linie der erhofften propagandistischen Wirkung. Die damit verbundenen deutschlandpolitischen Bezüge wurden aus dem Stalin-Befehl abgeleitet: Die Sowjetunion wolle Deutschland als Staat und das deutsche Volk als Nation nicht vernichten, aber nur die Erfüllung gewisser Grundbedingungen - Lossagen von Hitler, Räumung des besetzten Territoriums - ermögliche es Deutschland, den Krieg ohne Verlust seiner originären nationalen Interessen zu beenden ${ }^{68}$. In dem Rechenschaftsbericht des Büros für militärpolitische Propaganda - ebenfalls vom April 1942 - ging der Entwurf eines deutschen "Anti-Hitler-Komitees" noch einen Schritt weiter ${ }^{69}$. Im Vergleich zur ersten Vorlage hatte sich das Konzept insofern geändert, als nun die Bildung des Komitees aus Emigranten und Kriegsgefangenen vorgeschlagen wurde und es den Status einer Exilregierung bekommen sollte: „Ein solches Komitee, in dem Vertreter aller Klassen der deutschen Bevölkerung und Vertreter der Armee sein werden, wird auch die Regierung stellen, die einen für Deutschland würdigen Frieden schließen wird. Dieses Komitee rettet das Land vor der wirtschaftlichen und politischen Katastrophe und beseitigt das faschistische Regime."70

Parallel zu Manuil'skijs und Lozovskijs Vorstoß präsentierte die KPD ihrerseits einen Vorschlag zur Schaffung eines nationalen antifaschistischen Komitees. Eine Konferenz deutscher Volksvertreter - Exilkommunisten und Kriegsgefangene in der Sowjetunion - solle als Erwiderung auf den Tagesbefehl vom 23. Februar 1942 an Stalin das Angebot richten, sich für den Sturz Hitlers und die Beendigung des Krieges einzusetzen. In dem auf den 3. April 1942 datierten Entwurf hieß es weiterhin, auf der Konferenz solle ein Ausschuß zur Schaffung eines „Nationalkomitees“ gewählt werden, „das die Führung des Kampfes des deutschen Volkes für die Befreiung Deutschlands von der barbarischen Hitlerherrschaft und für die sofortige Beendigung des Krieges übernimmt“. Es wurden fünf Thesen aufgestellt: (1) „Zu-

67 Geschichte der deutschen Arbeiterbewegung, S. 558. Der Gedanke der Volksvertreter wurde bei der Gründung des NKFD aufgegriffen, indem alle Unterzeichner, insbesondere die wehrpflichtigen Soldaten und Reserveoffiziere mit ihrer Berufsbezeichnung und die Emigranten ohne Nennung der Parteimitgliedschaft aufgeführt wurden.

68 „Über einige Maßnahmen zur moralischen Zersetzung der deutschen Soldaten“ vom 3.4. 1942, RGASPI 495/77/30, Bl. 15-18.

69 Der Bericht ist nicht datiert, aber am 8.5. 1942 von Dimitrov abgezeichnet worden (RGASPI 495/77/14, Bl. 41). Bereits am 26.4.42 schrieb Burcev an Manuil'skij, daß er den Bericht auf der Sitzung des Büros für militärpolitische Propaganda am 27. 4. 1942 thesenartig vorstellen werde (RGASPI 495/77/14, Bl. 1).

70 „Bericht über die Propaganda unter den Truppen des Gegners während der 10 Monate des Vaterländischen Krieges“, RGASPI 495/77/17, Bl. 59. 
sammenschluß aller ehrlichen Deutschen zum gemeinsamen Kampf für den Sturz des blutigen Kriegstreibers und Volksfeindes Hitler“, (2) „Beendigung des Krieges (...) durch Sabotage, Streiks, Demonstrationen, politische Massenstreiks und bewaffneten Kampf bis zur Volkserhebung“, (3) „Abschluß eines Friedens“, (4) „Deutschland wird eine Volksrepublik“, (5) „Die Wirtschaft wird in den Dienst

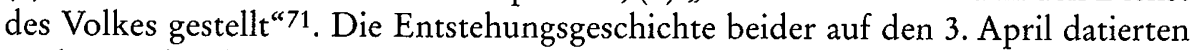
Vorlagen, der des Büros für militärpolitische Propaganda und der der KPD, läßt sich nicht zweifelsfrei klären. Vermutlich übernahmen Manuil'skij und Lozovskij, möglicherweise nachdem sie die deutschen Kommunisten mit der Ausarbeitung beauftragt hatten, die Idee eines nationalen Komitees aus Emigranten und Kriegsgefangenen, das den Widerstand gegen Hitler organisiert und für die Herbeiführung eines Friedens sorgt. Die politischen Absichten der KPD ließen sie weg und argumentierten taktisch sehr viel klüger mit den Möglichkeiten, die ein solches Komitee für die sowjetische Propaganda eröffne ${ }^{72}$.

Der Zeitpunkt, zu dem Manuil'skij und Lozovskij ihre Vorschläge unterbreiteten, war passend gewählt. Sie fielen in eine Zeit, in der die Leitung der Politischen Hauptverwaltung wechselte und infolgedessen das Büro für militärpolitische Propaganda umstrukturiert wurde. Ob der Vorstoß durch die Umbruchsituation motiviert war, oder ob lediglich versucht wurde, innerhalb des vorgegebenen politischen Rahmens - dem Stalin-Befehl - Handlungsspielräume zu nutzen, kann eindeutig nicht beantwortet werden. Ebenso kann aufgrund der dürftigen Quellenlage die Haltung der sowjetischen Führung zu den Vorschlägen lediglich an äußerlichen Veränderungen in der Politik der Politischen Hauptverwaltung abgelesen werden. Erst Anfang Mai 1942 wies Ščerbakov in einer Besprechung mit leitenden Mitarbeitern der 7. Verwaltung die Vorschläge zur Bildung eines Komitees aus Deutschen zurück ${ }^{73}$. Sinngemäß soll er festgelegt haben, daß deutsche Emigranten und kooperationswillige Kriegsgefangene stärker in die Propagandaarbeit eingebunden werden könnten, daß die Propaganda aber weiterhin ausschließlich von der Roten Armee - d.h. nicht von einem deutschen Komitee - geführt werde ${ }^{74}$. Auch auf der

71 Geschichte der deutschen Arbeiterbewegung Bd. 5, S. 559 ff.; zur Einbettung des dort nicht vollständig edierten Dokuments siehe „Nach Hitler kommen wir“, S. 37.

72 Auf die von Erler/Laude/Wilke („Nach Hitler kommen wir“, S. 38) geäußerte Vermutung, daß Stalin die Vorlage der KPD wegen der darin gemachten Nachkriegspläne abgelehnt habe, läßt sich somit entgegnen, daß die Vorlage der KPD nie Gegenstand der Beratung wurde, sondern nur in der von Manuil'skij und Lozovskij ausgearbeiteten Variante Eingang in die Diskussion der sowjetischen Entscheidungsträger fand. Vgl. Fischers (Sowjetische Deutschlandpolitik, S. $49 \mathrm{ff}$.) Einordnung des KPD-Vorschlages, die jedoch nichts über die sowjetische Haltung aussagt.

73 Burcev schildert in seinen Erinnerungen (Einsichten, S. 86) die Kurskorrektur mit den Worten: „Ich muß gestehen, daß wir die ersten Ergebnisse der ideologischen Einwirkung auf die gegnerischen Truppen nicht ganz nüchtern bewertet hatten. Die erbeuteten Dokumente, die (...) Befehle und Briefe hatten uns zu dem Schluß verleitet, daß ein erheblicher Teil der Wehrmachtsangehörigen für unsere Propaganda schon aufnahmebereit geworden sei. Das war aber nicht der Fall, und das Zentralkomitee mußte uns korrigieren."

74 Ebenda, S. 86: „,Eine Demoralisierung der deutschen Wehrmacht ist nicht zu erkennen“, faßte Ščerbakov seine Eindrücke von dem Vortrag zusammen. (...), Wir müssen die deutschen politischen Emigranten verstärkt in unsere Arbeit einbeziehen, um so mehr, als sie darauf brennen. Das gleiche trifft auf die Kriegsgefangenen zu, die sich zur Mitarbeit bereit 
Sitzung des neu gebildeten Rates für militärpolitische Propaganda am 27. Juni 1942 betonte Ščerbakov demonstrativ die Konzentration auf die eigenen propagandistischen Kräfte des GlavPURKKA: In der Propaganda sei es wichtig, die Furcht der deutschen Soldaten vor der militärischen Stärke der Sowjetunion und der der Westalliierten zu schüren, die Ängste der deutschen Soldaten (Sorge um die Familie, Heimweh usw.) propagandistisch zu nutzen und die Differenzen innerhalb der Wehrmacht und der mit ihr verbündeten Armeen zu verstärken. Von einer Zusammenarbeit mit Deutschen - Emigranten oder Kriegsgefangene - sprach Ščerbakov überhaupt nicht. Vielleicht wollte er sie in Aussicht stellen, wenn er sagte: „Nach einiger Zeit, abhängig von der Möglichkeit einer Veränderung der Lage, können neue Punkte aufgestellt werden." 75

Im Frühjahr 1942 wurde die Idee eines antifaschistischen deutschen Komitees zur propagandistischen Unterstützung der Roten Armee von der sowjetischen Führung abgelehnt. Die Rote Armee vertraute auf ein starkes Auftreten und wollte nicht öffentlich mit deutschen Emigranten oder Kriegsgefangenen auf sowjetischem Boden kooperieren. Die Gestaltung der politischen Arbeit unter den Kriegsgefangenen durch die Komintern war daher eine elegante Lösung, mit den deutschen Kommunisten - und Kriegsgefangenen - dennoch zu einer Zusammenarbeit zu kommen.

\section{Kommission der Komintern}

Die Komintern blieb bis zu ihrer Auflösung personell die tragende Säule der politischen Arbeit in den Kriegsgefangenenlagern. Im Januar 1942 beschloß das EKKI auf der Grundlage der Delegationsberichte aus den Lagern Temnikov und SpasoZavodsk, in den Lagern des UPVI eine „ständige konkrete Aufklärungsarbeit in bezug auf die Sowjetunion als sozialistischer Arbeiter- und Bauernstaat" und „eine populäre Propaganda unserer kommunistischen Grundsätze“ $z u$ führen ${ }^{76}$. Nun wurde dauerhaft eine Kommission "zur ständigen Bearbeitung der politischen Fragen der Kriegsgefangenenarbeit" eingesetzt. Ihre Mitglieder waren Johann Koplenig (KPÖ), Béla Szántó (KPU) und Walter Ulbricht (KPD), der den Vorsitz führte ${ }^{77}$. Als Aufgaben wurden festgesetzt: (1) Schaffung eines Schulungslagers, (2) Herausgabe eines Informationsbulletins, (3) Ausarbeitung von Aufrufen, (4) Instruktions-, Vortrags- und Studienreisen in die Lager und (5) die Herausgabe von antifaschistischen Flugschriften und Broschüren für die Kriegsgefangenen in verschiedenen Sprachen ${ }^{78}$. Die Kommission nahm ihre Arbeit am 31. Januar 1942 auf.

erklärt haben. Nur dürfen wir ihre Aktion gegen Krieg und Faschismus nicht mit der Propaganda unserer Politorgane gleichsetzen." “

75 Protokoll der zweiten Sitzung des Rates für militärpolitische Propaganda vom 27. 6. 1942, RGASPI 88/1/947, Bl. 6.

76 „Kurze Richtlinien für die Arbeit unter den Kriegsgefangenen“ (Anlage zu dem Protokoll der EKKI-Sitzung vom 24. 1. 1942), RGASPI 495/77/30, Bl. 13-14.

77 Beschlußprotokoll der EKKI-Sitzung ,wegen des Berichtes der Arbeit im Kriegsgefangenenlager Karaganda" vom 24. 1. 1942, ebenda, Bl. 10-12.

78 „Einzelvorschläge für die Kommission zur Behandlung der Kriegsgefangenenfragen“ (Anlage zu dem EKKI-Protokoll vom 24. 1. 1942), RGASPI 495/77/4, Bl. 16-18. 
Man beschloß die „Kampagne für Zustimmungserklärungen von Kriegsgefangenen zu den Aufrufen für einzelne Länder" weiterzuführen ${ }^{79}$. Diese Zustimmungserklärungen bezogen sich - für die deutschen Kriegsgefangenen - auf den im Oktober $1941 \mathrm{im}$ Lager Temnikov verabschiedeten „Appell der 158“. In den nun folgenden Monaten wurden auch in anderen Lagern sogenannte Konferenzen abgehalten, auf denen sich die Kriegsgefangenen per Unterschrift - sogenannte Anschlußerklärungen - den Forderungen des "Appells der 158“ anschließen sollten ${ }^{80}$.

Eine der wesentlichen Aufgaben der EKKI-Kommission war neben der Ausarbeitung von Appellen und Propagandamaterial die Organisation eines Schulungslagers für Kriegsgefangene. Die Kommission konzipierte eine viermonatige Schulung für 120 deutsche, 15 österreichische, 10 sudetendeutsche und 80 rumänische Kriegsgefangene in einer sogenannten Antifa-Schule. Sobald mehr Kriegsgefangene anderer Nationen zur Verfügung stehen würden, sollten die Schulungen ausgeweitet werden. Die Schule sollte unter der technischen Verwaltung des UPVI stehen, der Schulleiter selbst aber „ein Propagandist“, d.h. Politoffizier des GlavPURKKA sein. Als Lehrer schlug die Kommission Funktionäre der KPD und KPR vor, und für die Behandlung aktueller politischer Themen sollten leitende Funktionäre der kommunistischen Parteien als Lektoren hinzugezogen werden ${ }^{81}$. Die erste AntifaSchule wurde in dem Kloster Oranki, $60 \mathrm{~km}$ südlich von Nižnij Novgorod (Gor'kij), eingerichtet, das zu einem Kriegsgefangenenlager (Lager Nr. 74) umfunktioniert worden war. Leiter der Antifa-Schule wurde der Offizier der 7. Verwaltung des GlavPURKKA Nikolaj Janzen². Der erste Kurs begann am 10. Mai 1942 und dauerte drei Monate ${ }^{83}$. Ihn absolvierten 71 deutsche (inklusive Österreicher und Sudetendeutsche) und 22 rumänische Kriegsgefangene. Von Oktober bis Dezember 1942 folgte der zweite Kurs ${ }^{84}$.

Im Sommer 1943 wurde eine zweite Antifa-Schule gegründet. Nach Abschluß des zweiten Durchlaufes an der ersten Schule in Oranki hatte der Schulleiter Janzen zu bedenken gegeben, daß „ein bedeutender Teil der Schulabsolventen bisher nicht die notwendige politische Standhaftigkeit, ideologische Klarheit und gebührende moralische Festigkeit“ erreicht habe. Er schlug daher die Einrichtung einer „speziellen Lager-Schule" vor, um für die Antifa-Schule eine bessere Vorauswahl treffen zu können ${ }^{85}$. Diese Lager-Schule wurde am 19. Januar 1943 vom EKKI beschlossen $^{86}$. Kurz darauf, nach dem sowjetischen Sieg bei Stalingrad, wurde der Beschluß

79 Protokoll der EKKI-Kommission vom 31. 1. 1942, RGASPI 495/77/5, Bl. 27.

80 Robel, Antifa, S. 38 ff. Zur Programmatik siehe auch Sywottek, Deutsche Volksdemokratie,

S. $115 \mathrm{ff}$.; Fischer, Sowjetische Deutschlandpolitik, S. $22 \mathrm{ff}$.

81 Protokoll der EKKI-Kommission vom 13. 2. 1942, RGASPI 495/77/17, Bl. 4-5.

82 Jancen war, bevor er von der 7. Verwaltung mobilisiert wurde, Philosophieprofessor an der Leningrader Universität. Nach dem Krieg lehrte er an der Parteihochschule der KPdSU. Er war Baltendeutscher. Im folgenden wird für seinen Namen die deutsche Schreibweise "Janzen" verwendet; Fey, Ein Totgesagter kehrt zurück, S. 285 ff.; Hoffmann, Moskau Berlin, S. 63 ff.; vgl. Robel, Antifa, S. 257 f.

83 Janzen an Dimitrov am 10. 8. 1942, RGASPI 495/77/20, Bl. 54; Abschlußbericht Janzens über den ersten Kurs vom 17. 8. 1942, ebenda, Bl. 124; vgl. Robel, Antifa, S. 200.

84 Siehe S. 132.

85 Bericht über den 2. Kurs an der Antifa-Schule Oranki, Janzen an Dimitrov am 16. 1. 1943, RGASPI 495/77/26, Bl. 8 RS.

86 „Beschluß des EKKI-Sekretariats zum Bericht des Direktors der antifaschistischen Polit- 
revidiert und die Lager-Schule zu einer regulären zweiten Antifa-Schule aufgewertet. Diese sollte als „Massenschule“ mit 1000 bis 1500 Schülern aufgebaut werden, die in Kursen von vier bis sechs Wochen Dauer zu Lageraktivisten, d.h. Brigadiere in Arbeitskommandos, ausbildet würden. Die erste Antifa-Schule dagegen sollte bei einer Größe von 250 bis 300 Schülern bleiben und diese in dreimonatigen Kursen zu Instrukteuren und leitenden Politarbeitern in den Lagern schulen ${ }^{87}$. Durch die unterschiedliche Aufgabenstellung der Schulen kam es zu einer Zweiteilung des Schulungssystems. Das konkrete Ausbildungsziel blieb an beiden Schulen der Einsatz als Lagerpropagandist, aber durch die unterschiedlich intensive Schulung und die daraus resultierende unterschiedliche "politische Reife“ gab es nun eine Ausdifferenzierung der Absolventen. Daraus konnten - je nach Bedarf - weitere Verwendungsmöglichkeiten abgeleitet werden.

Die Rolle der EKKI-Kommission bei der Organisation der Antifa-Schulen beschränkte sich auf die Zuarbeit. Nominell fällte sie zwar die Beschlüsse, mußte sich diese jedoch immer vom EKKI bestätigen lassen, das in seiner Entscheidung wiederum auf die Zustimmung der maßgeblichen sowjetischen Funktionäre angewiesen war. Bevor die Kommission ihre eigentliche Arbeit aufnahm, versicherte sich daher das EKKI des Rückhalts bei den Verwaltungsspitzen im ZK der VKP (b). Dimitrov bat Ščerbakov, ihn bei der Durchsetzung der von der EKKI-Kommission gefaßten Beschlüsse gegenüber NKVD und GlavPURKKA zu unterstützen ${ }^{88}$. An den NKVD-Chef Berija richtete er ein inhaltlich identisches Schreiben, wobei er deutlich auf bestehende Abstimmungsprobleme hinwies: „Da sich mit den Fragen der politischen Arbeit unter den Kriegsgefangenen unterschiedliche Organe beschäftigen und nicht immer die notwendige Übereinstimmung und Umsicht erzielt werden kann, haben wir Genossen Ščerbakov gebeten, daß die laufende Arbeit einem geeigneten Mitarbeiter in der Propagandaabteilung des ZK der VKP (b) übertragen wird, damit dieser sich ständig mit den Fragen dieser Arbeit befaßt und in Verbindung mit der Verwaltung für die Angelegenheiten von Kriegsgefangenen des NKVD der UdSSR und mit uns steht."89 Dimitrovs Wünschen wurde stattgegeben. Am 12. Februar bestätigte Georgij Aleksandrov, Leiter der Propagandaabteilung, daß im ZK ein Verantwortlicher für die Arbeit unter den Kriegsgefangenen benannt worden sei. Einen Tag darauf gab Ščerbakov seine Zustimmung ${ }^{90}$. Typisch für die Abläufe in der sowjetischen ZK-Bürokratie war, daß beide Spitzenfunktionäre ihre Entscheidung mündlich am Telefon mitteilten, Dimitrov also über keine schriftliche Bestätigung verfügte. Besprechungen bzw. Telefonate waren elementarer Bestandteil bei der Befehlsübermittlung.

Das Problem der Koordination blieb für die gesamte Dauer der Tätigkeit der

schule Genosse Janzen über die Ergebnisse der Arbeit des zweiten Kurses der Schule" vom 19. 1. 1943, RGASPI 17/125/183, Bl. 5-6.

87 Ebenda.

88 Dimitrov an Ščerbakov am 2. 2. 1942, RGASPI 495/77/33, Bl. 6.

89 RGASPI 495/77/21, Bl. 1. Anhand der handschriftlichen Bemerkungen auf dem Brief wird deutlich, daß Berija den Brief weiterreichte an seinen für das UPVI zuständigen Stellvertreter Serov, der ihn weiterleitete an UPVI-Chef Soprunenko.

90 Dimitroff, Tagebücher, Einträge vom 12. 2. und 13. 2. 1942; danach wurde Kondakov, stellvertretender Leiter der Presseabteilung im ZK, zum Verantwortlichen für die Arbeit unter den Kriegsgefangenen ernannt. Für die laufende Arbeit war es offenbar Tukarinov, über dessen genaue Funktion im ZK-Apparat aber nichts bekannt ist. 
EKKI-Kommission das entscheidende Hindernis. Bereits der Zustimmung Dimitrovs zu den Vorschlägen der EKKI-Kommission über die Gestaltung der AntifaSchulung, d. h. ihrem eigentlichen Auftrag, mußten nach dem oben dargelegten Mechanismus Absprachen auf höherer Ebene vorausgehen ${ }^{91}$. Im April 1942, die Kommission hatte gerade ihre Arbeit aufgenommen, beklagte sich Ulbricht gegenüber Dimitrov, daß faktisch keine Verbindung zwischen den Kriegsgefangenenlagern und der EKKI-Kommission bestehe. Man sei von aktuellen Informationen abgeschnitten, denn alles Material werde direkt an die 7. Verwaltung oder die Propagandaabteilung des ZK der VKP (b) geschickt. Ulbricht konstatierte: „Gegenwärtig beschäftigen sich vier Stellen mit der politischen Arbeit unter den Kriegsgefangenen: 1. In der Hauptverwaltung der Kriegsgefangenenlager-Genosse Worobjew [Vorob'ev], 2. die Redaktion der Kriegsgefangenenzeitung, die der 7. Abteilung untersteht, 3. in der Propagandaabteilung des ZK bearbeitet diese Fragen Genosse Tukarinow, 4. bearbeiten wir im Kominternapparat diese Fragen. (...) Notwendig wäre, daß bei bestimmten politischen Anlässen, wie z.B. nach dem Stalin-Befehl vom 1. Mai eine gemeinsame Besprechung der Genossen stattfindet, die sich mit der politischen Arbeit unter den Kriegsgefangenen beschäftigen. " ${ }^{22}$ Mit Tukarinov von der Propagandaabteilung traf sich Ulbricht im Mai. Zusammen arbeiteten sie „Vorschläge für die Verbesserung der Arbeit unter den Kriegsgefangenen" aus, die dem Leiter der Propagandaabteilung, Aleksandrov, im Juni 1942 unterbreitet wurden ${ }^{93}$. Doch eine Verbesserung der Koordination wurde dadurch nicht erreicht. Anfang Juli 1942 beschloß die EKKI-Kommission abermals: „Sinnvoll wären regelmäßige Treffen mit Tukarinov (ZK VKP), Vorob'ev (UPVI), Ulbricht und Szántó und einem Vertreter der PUR [GlavPURKKA]." ${ }^{49}$ Das Problem der gegenseitigen Abstimmung blieb dennoch bestehen.

Ein weiteres Problem der EKKI-Kommission waren die „Klagen der Kommissare in den Lagern, daß keine Antworten auf ihre Fragen und keine Beurteilung des von ihnen geschickten Materials erfolgt ${ }^{\text {" } 95}$. Für diese Beschwerden der Politinstrukteure allerdings war die Kommission selbst verantwortlich, denn lange Zeit war auf Anfragen nicht reagiert worden. Erst nachdem zahlreiche Klagen aus den Lagern eingegangen waren, sah sich Ulbricht genötigt, Dimitrov zum Handeln zu bewegen: „Anliegend übermittle ich Ihnen weiter den Brief mit den Wünschen unserer Genossen aus dem Lager Spassko-Sawodsk [Spaso-Zavodsk] und unsere Antwort. Bisher haben wir auf solche Briefe nicht geantwortet. Es erscheint uns aber doch notwendig, den Genossen auf dem Weg über die Hauptverwaltung [UPVI]

91 Vgl. ebenda, Einträge vom 18. 2. und 25. 2. 1942; Protokoll der EKKI-Kommission vom 5. 3. 1942, RGASPI 495/77/17, Bl. 15.

92 Ulbricht an Dimitrov, RGASPI 495/77/17, Bl. 31+RS. Der Brief ist nicht datiert. Er ist aber, der Reihenfolge in der Akte nach zu schließen, vermutlich im April 1942 geschrieben worden.

93 „Vorschläge für die Verbesserung der Arbeit unter den Kriegsgefangenen“ vom 20. 5. 1942, RGASPI 495/77/16, Bl. 2-5. Die Vorschläge waren in der Anlage zu dem Brief Ulbrichts an Aleksandrov, in dem er auf die Absprache mit Tukarinov verweist. Die hier zitierte Kopie des Briefes trägt kein Datum, ist aber der Reihenfolge in der Akte nach zu schließen vom Juni 1942; Text ediert bei Uhlig, Rückkehr aus der Sowjetunion, S. 163.

94 Protokoll der EKKI-Kommission vom 2. 7. 1942, RGASPI 495/77/16, Bl. 92.

95 Ebenda. 
kurz zu antworten. "96 An diesen Zeilen wird das grundsätzliche Problem der Koordinationsversuche in der politischen Arbeit deutlich. Die Kommunikationswege der handelnden Funktionäre waren an die Hierarchie innerhalb der Apparate gebunden. Die vom EKKI in die Lager entsandten Politinstrukteure waren nur über die Verwaltung der Kriegsgefangenenlager zu erreichen, wobei das UPVI wenig Interesse an einer Zusammenarbeit mit der EKKI-Kommission zeigte. Erschwerend kam hinzu, daß sich bei inhaltlichen Entscheidungen die untergeordnete Instanz immer bei der nächst höheren rückversichern mußte. Sowohl die Entscheidungsfindung wie auch die Umsetzung der Beschlüsse verlangsamten sich dadurch enorm.

Eigene inhaltliche Akzente setzte die EKKI-Kommission nicht. Vielmehr lagen die grundsätzlichen Entscheidungen in der politischen Arbeit unter den Kriegsgefangenen weiterhin bei Dimitrov bzw. Manuil'skij. Besonders deutlich wurde das bei der Ausweitung der Antifa auf alle Dienstgrade, d.h. der Einbeziehung der Offiziere durch die Gründung einer „antifaschistischen Offiziersgruppe“. Im Herbst 1941 hatte „Professor Arnold“ begonnen, Gespräche mit einzelnen Offizieren zu führen mit dem Erfolg, daß sich im November 1941 die Offiziere Ernst Hadermann, Friedrich Reyher, Eberhard Charisius und Joachim Sagasser von Hitler lossagten. Ihre Distanzierung vom Nationalsozialismus wurde aber auf den Rat Arnolds nicht öffentlich bekannt gegeben, damit sie das Vertrauen ihrer Offizierskameraden nicht verloren. Erst als sich im April 1942 Leutnant Friedrich Augustin dieser Gruppe anschloß, änderte sich die Situatuion. Augustin, der später vom sowjetischen Geheimdienst angeworben und im Februar 1944 von der Gestapo verhaftet wurde 97 , schilderte in einem Verhör sein Auftreten in der Offiziersgruppe: „Nachdem ich diese [antifaschistische] Überzeugung gewonnen hatte, erklärte ich das gegen den Willen von Maria [eine Politinstrukteurin] öffentlich vor allen deutschen Offizieren. Dies bewirkte, daß die vier oben erwähnten Offiziere, die ja schon seit mehreren Monaten gegen Adolf Hitler waren, aus dem Dunkel hervortraten, ebenfalls ihre neue Gesinnung offenbarten und wir zu fünft nun zusammen mit Gonzales, Maria und Wagner [drei Politinstrukteure] die Propaganda unter den deutschen Offizieren betrieben." 98 Angesichts dieser Rekonstruktion der Ereignisse ist die Einschätzung Wolffs, die Offiziere hätten „erste Anzeichen zur Mitarbeit gezeigt“, woraus sich dann die Konstituierung der antifaschistischen Offiziersgruppe entwickelt habe, ein Euphemismus. Allerdings hat seine weitere Schilderung durchaus Anspruch auf Glaubwürdigkeit: „Professor Arnold, der darüber vor sowjetischen und deutschen Mitarbeitern der Komintern in Ufa berichtet hatte, war von dort mit der Weisung zurückgekehrt, die Konstituierung dieser Offiziere zu

96 Ulbricht an Dimitrov am 23. 6. 1942, RGASPI 495/77/17, B1. 74.

97 Augustin durchlief nach der Antifa-Schulung eine sowjetische Agentenausbildung und wurde im April 1943 als Attentäter auf den in deutschen Diensten stehenden russischen General Vlassov in Berlin angesetzt. Er hielt sich mehrere Wochen in Berlin auf, führte seinen Auftrag aber nicht aus, worüber er bei der Rückkehr nach Moskau seine Auftraggeber täuschen konnte. Bei seinem zweiten Einsatz, dem geplanten Attentat auf den Kommandeur der Sicherheitspolizei in der Ukraine, von Gottberg, offenbarte er sich der deutschen Sicherheitspolizei, die ihn verhaftete und eingehend verhörte. Augustin wurde im Konzentrationslager Sachsenhausen hingerichtet; vgl. Robel, Antifa, S. 59, Anm. 262.

98 Bericht der deutschen Sicherheitspolizei über die Vernehmung Augustins vom 14. 2. 1944, Litauisches Zentrales Staatsarchiv 1399/1/39, Bl. 6 RS-7. Ich danke Christoph Dieckmann für diesen Hinweis. 
einer antifaschistischen Offiziersgruppe und ihr erstes offenes Auftreten im Lager vorzubereiten." 99

Die Kominternführung trat aufgrund der Panne im Offizierslager, worüber Arnold in Ufa berichtet haben wird, die Flucht nach vorne an und befürwortete für Außenstehende vollkommen überraschend - die Gründung einer AntifaGruppe für Offiziere. Es ist daher auch nicht erstaunlich, daß Manuil'skij erst die „Bedenken innerhalb der KPD-Führung gegen eine solche Erweiterung der bis dahin rein proletarischen Antifa" 100 ausräumen mußte, bevor es am 30./31. Mai 1942 zur „Ersten Konferenz antifaschistischer Offiziere“ kam. Angesichts der bisherigen Politik des EKKI, das sich in der Regel auf die inhaltliche Zuarbeit der KPD stützte, war das Zögern der deutschen Kommunisten zu verstehen. Es zeigt aber auch die Schwierigkeiten innerhalb des sowjetischen Apparates, auf unvorhergesehene Zwischenfälle zu reagieren. Während die Führungsfunktionäre Dimitrov und $\mathrm{Ma}$ nuil'skij schnell handeln konnten - wahrscheinlich in direkter Rücksprache mit der sowjetischen Führung -, waren die untergeordneten Funktionäre desorientiert. Erst nach einem Moment der Irritation schwenkte die KPD bzw. die EKKI-Kommission auf die eingeschlagene Linie ein: $\mathrm{Da}$ die Offizierskonferenz gezeigt habe, daß es ebenso unter den Offizieren „Antihitleristen“ gebe, sei es sinnvoll, auch diese antifaschistisch zu schulen ${ }^{101}$.

Die Aufrufe und Erklärungen der Antifa orientierten sich an der Programmatik der KPD bzw. der jeweiligen kommunistischen Partei, mußten aber immer mit dem EKKI vorher abgestimmt werden. Deutlich wird dieser Mechanismus an der Ausarbeitung des „Manifestes an das deutsche Volk und die deutsche Armee“ vom 6. Dezember 1942, das den „Appell der 158“ als programmatische Basis für die politische Arbeit unter den deutschen Kriegsgefangenen ablöste. Nach vorheriger Abstimmung mit Dimitrov beauftragte die KPD Anton Ackermann mit der Ausformulierung eines ersten Entwurfes. Dieser wurde von einer eigens eingesetzten Kommission des EKKI bearbeitet, die den Entwurf zusammen mit weitreichenden Auflagen zur Überarbeitung an die KPD zurückreichte. Eine daraufhin von der KPD vorgelegte zweite Variante wurde nochmals einer eingehenden Schlußredaktion unterzogen, dieses Mal unter Beteiligung von Generalsekretär Dimitrov und Manuil'skij in seiner Eigenschaft als Mitglied des Rates für militärpolitische Propaganda ${ }^{102}$. Schließlich folgte der Beschluß des EKKI, „die von den deutschen Genossen ausgearbeitete Linie und die Linie des von der Kommission behandelten ,Manifestes an das deutsche Volk und die deutsche Armee` im grundlegenden zu billigen" 103 .

Im „Friedensmanifest des deutschen Volkes“ tauchten einzelne Versatzstücke des Komitee-Vorschlages vom Frühjahr 1942 wieder auf. Eine Konferenz „verantwortungsbewußter Deutscher", die sich „ungeachtet des Glaubens und der politischen Richtung" zu einer "geheimen Beratung" zusammengefunden hätten, stellte ein zehn Punkte umfassendes Aktionsprogramm auf. Die ersten beiden Forderungen

99 Wolff, Die erste Konferenz, S. 279.

100 Robel, Antifa, S. 54.

101 Protokoll der EKKI-Kommission vom 22. 6. 1942, RGASPI 495/77/16, Bl. 87.

102 „Nach Hitler kommen wir", S. 46f.; vgl. Sassning, Genesis und Bedeutung des Friedensmanifestes, S. $789 \mathrm{ff}$; vgl. Dimitroff, Tagebücher, Einträge vom 1.12., 4. 12., 8. 12., 9. 12. und 10. 12. 1942.

103 EKKI-Protokoll Nr. 812 vom 15. 12. 1942, SAPMO-BArch NY 4036/542, Bl. 70. 
waren „sofortige Einstellung der Kriegshandlungen“ sowie „Sturz der Hitlerregierung und die Schaffung einer nationalen demokratischen Friedensregierung". Weiterhin wurden Pressefreiheit, Aufhebung der nationalsozialistischen Gesetzgebung, Arbeit, Sozialfürsorge und eine friedliche Außenpolitik gefordert ${ }^{104}$. Die Friedenskonferenz war frei erfunden. Weder fand sie im Westen Deutschlands statt, noch waren irgendwelche Vertreter des deutschen Volkes versammelt, sondern es sprachen ausgewählte Sprecher nach sorgfältig ausformulierten Manuskripten über den von der KPD in Moskau - unter der Leitung der Komintern - betriebenen deutschen Volkssender ${ }^{105}$. Bemerkenswert war dennoch der Konzeptionswechsel der KPD, der mit dem vom EKKI diktierten Manifest verbunden war ${ }^{106}$.

Die Idee einer alle Schichten umfassenden Bewegung und die Zurückstellung eines kommunistischen Programms führten innerhalb der KPD zu Irritationen. In einem Brief an den in einem Kriegsgefangenenlager als Politinstrukteur arbeitenden Gottfried Keller versuchte Wilhelm Pieck die neue Parteilinie zu erklären: „Es gibt zwei Wege, dem Krieg ein Ende zu machen. Die militärische Niederlage und die Volkserhebung zum Sturz Hitlers. Wir ziehen selbstverständlich den letzteren Weg vor, weil damit das deutsche Volk nicht nur den Krieg abkürzt, sondern sich auch wieder Achtung und Ansehen bei den anderen Völkern erwirkt und einen gerechten Frieden sich verschaffen wird. Das ist die eine Hauptaufgabe. Die andere Hauptaufgabe ist: Was soll nach Hitler kommen? Dafür gibt das Friedensmanifest ausreichend Auskunft. Wir wollen also ein demokratisches Deutschland an Stelle des Hitlerregimes. Wir erheben die Forderung deswegen, weil nicht nur auf dieser Basis die breite Kampffront geschaffen werden kann, sondern weil wir einen solchen $\mathrm{Zu}$ stand brauchen, um den Massen die Möglichkeit zu verschaffen, auf dem Boden der Demokratie ihren Einfluß geltend zu machen und sich politisch zu orientieren. Erst dann werden die Massen entscheiden können, welchen weiteren Weg sie in der Entwicklung des Staates und der Wirtschaft gehen wollen."107 Die KPD schwenkte auf die vorgegebene Linie ein, daß im Mittelpunkt jeder Propaganda die Beendigung des Krieges stehen müsse und demgegenüber die Vorstellungen der KPD von einem sozialistischen Nachkriegsdeutschland zurückzutreten haben. So wie die fiktive "westdeutsche Friedenskonferenz" alle 'Teile der Bevölkerung des Deutschen Reiches mobilisieren sollte, so sollten auch in den sowjetischen Kriegsgefangenenlagern alle Dienstgrade in die politische Arbeit einbezogen werden. In diesem Sinne erläuterte das UPVI den Lagerleitungen das „Friedensmanifest“ in einem Rundschreiben: „Die grundlegende Aufgabe besteht darin, unter den deutschen Kriegsgefangenen eine Massenbewegung zur Unterstützung des in Deutschland beginnenden Kampfes für den Frieden zu initiieren. Dem Programm der aktiven westdeutschen Friedensbewegung muß besondere Aufmerksamkeit gewidmet werden. Dieses Programm gibt eine konkrete Vorstellung von dem neuen Deutschland nach der

104 „Friedensmanifest an das deutsche Volk und an die deutsche Wehrmacht der westdeutschen Beratung der nationalen Friedensbewegung vom 6. Dezember 1942“, in: Zur Geschichte der deutschen antifaschistischen Widerstandsbewegung, S. 179.

105 „Nach Hitler kommen wir", S. 49

106 Dazu siehe Sywottek, Deutsche Volksdemokratie, S. 119 ff.; Fischer, Sowjetische Deutschlandpolitik, S. 52; Robel, Antifa, S. $61 \mathrm{f}$.

107 Pieck an Keller am 13. 1. 1942; RGASPI 495/18/1339a, Bl. 14. 
Niederwerfung des Hitlerregimes und ermöglicht es, alle Schichten der Kriegsgefangenen in den aktiven Kampf der Friedensbewegung einzubeziehen. "108

Das Konstrukt einer „westdeutschen Friedenskonferenz" bediente sich der üblichen Organisationsformen der politischen Arbeit unter den Kriegsgefangenen. Sie war vergleichbar mit den Kriegsgefangenenkonferenzen zur Verabschiedung vorformulierter Aufrufe, wie sie im Frühjahr 1942 als sogenannte Anschlußerklärungen an den "Appell der 158“ durchgeführt worden waren. So gesehen war die "westdeutsche Friedensbewegung" nicht vergleichbar mit dem späteren Nationalkomitee, das ohne konspirative Verschleierung mit offizieller sowjetischer Unterstützung gegründet wurde. Dennoch markierte das „Friedensmanifest“ eine weitere Etappe in der Entwicklung, die mit dem Vorstoß zur Gründung eines „Anti-HitlerKomitees“ im Frühjahr 1942 von Manuil'skij eingeleitet worden war. Es zeigte, daß die sowjetische Führung bereit war, auf kommunistische Vorstellungen zu verzichten, wenn es denn zu einem schnellen Ende des Krieges führen würde ${ }^{109}$.

Im Februar 1943 wurde die EKKI-Kommission personell aufgestockt. Hintergrund war der große Zustrom an neuen Kriegsgefangenen nach dem sowjetischen Sieg bei Stalingrad und die damit verbundene Ausweitung der politischen Arbeit in den Lagern. Ulbricht führte nach wie vor den Vorsitz. Aufgeteilt auf die Mitglieder eines sogenannten Arbeitsausschusses sollte die Kommission in Verbindung mit UPVI und GlavPURKKA die politische Arbeit in den Lagern sowie die Arbeit der - vom GlavPURKKA herausgegebenen - Kriegsgefangenenzeitung überwachen (verantwortlich: Béla Szántó) und die Antifa-Schule betreuen (verantwortlich: Paul Försterling). Ohne Nennung eines konkreten Aufgabengebietes wurde Ivanjuk vermutlich für die Arbeit unter den rumänischen Kriegsgefangenen - und Edwin Hoernle ebenfalls ohne nähere Aufgabenbeschreibung als „Referent“ hinzugeholt. Die restlichen Mitglieder der erweiterten EKKI-Kommission, die nicht im Arbeitsausschuß vertreten waren, fungierten als Vertreter der KPU (Mátyás Rákosi), der KPÖ (Johann Koplenig), der KPR (Draganov) und des EKKI (Belov als Leiter der Kaderabteilung und Ivan Morozov als Leiter des Schulungssektors des EKKI) ${ }^{110}$. Bis zu ihrer Auflösung tagte die EKKI-Kommission vorwiegend in der Zusammensetzung Ulbricht, Försterling, Ivanjuk und Hoernle.

Aber auch nach der personellen Erweiterung der EKKI-Kommission änderte sich nichts an den grundlegenden Problemen. Es fehlte die Zusammenarbeit der maßgeblichen Instanzen. Der Vorschlag des EKKI lautete daher: „Zur erfolgreichen Leitung der Arbeit unter den Kriegsgefangenen wird es für zweckmäßig erachtet, ein einheitliches Leitungszentrum zu schaffen, das bei der Politischen Hauptverwaltung angesiedelt ist und sich aus Vertretern der Politischen Hauptverwaltung,

108 Rundschreiben des UPVI an die Lagerleitungen vom 16. 1. 1943, RGVA/K 4p/3/1, Bl. 5.

109 Vgl. Firsov, Die sowjetische Deutschlandplanung, S. $127 \mathrm{ff}$. Firsov ordnet den Brief Dimitrovs an Keller nicht ganz zutreffend ein, indem er ihn als Antwort Dimitrovs auf die Anfrage eines Kriegsgefangenen interpretiert. Zudem diskutiert er ihn im Zusammenhang mit der Frage eines deutschen Freikorps; ein Gedanke, der in dem Brief zwar thematisiert wird, aber meines Erachtens nicht der entscheidende ist. Da die Aufstellung eines Freikorps aus deutschen Kriegsgefangenen nicht ernsthaft zur Debatte stand, kommt Firsov zu dem Schluß, auch das Nationalkomitee sei immer nur ein sowjetischer Propagandatrick gewesen.

110 EKKI-Beschluß vom 5. 2. 1943, RGASPI 495/77/26, Bl. 25. 
der Propagandaabteilung des ZK der VKP (b), der Verwaltung der Kriegsgefangenenlager und der EKKI-Kommission für die Arbeit unter den Kriegsgefangenen zusammensetzt." 111 Die Propagandaabteilung des ZK der VKP (b), die am 12. Februar vom EKKI benachrichtigt wurde, setzte daraufhin eine Besprechung an „zur Realisierung der Beschlüsse"112. Vermutlich ist es jedoch nicht mehr zu dieser Besprechung gekommen. Am 24. April 1943 einigten sich Politische Hauptverwaltung (Ščrbakov, Manuil'skij), Propagandaabteilung des ZK der VKP (b) (Aleksandrov), NKVD (Kruglov) und UPVI (Petrov) darauf, daß die politische Arbeit in den Kriegsgefangenenlagern allein vom NKVD - d.h. UPVI - durchgeführt werde. Die Koordinierung und alleinige Weisungsbefugnis sollte beim ZK der VKP (b) liegen: „Die Verwaltung des NKVD für Angelegenheiten der Kriegsgefangenen darf ihre Anweisungen nur über das ZK erhalten. Von der unmittelbaren Einbeziehung des EKKI ist abzusehen, und im weiteren ist diese Verbindung über die 7. Verwaltung des GlavPURKKA herzustellen. "113 Diese Besprechung stellt den Schlußpunkt der Mitwirkung der Komintern an der politischen Arbeit unter den Kriegsgefangenen dar. Die sowjetische Führung reagierte mit dieser Entscheidung auf die dauernden Koordinierungsprobleme, die eine effektive Arbeit behinderten. Allerdings wurden die konkreten Organisationstrukturen erst nach Auflösung der Komintern festgelegt, so daß das UPVI dann doch nicht die bereits zugesicherten Kompetenzen erhielt, sondern diese an das Institut 99 gingen. Personell blieben die ausländischen Kommunistischen Parteien auch weiterhin an der politischen Arbeit in den Kriegsgefangenenlagern beteiligt. Ulbricht, ehemals Leiter der EKKI-Kommission, wurde Mitarbeiter im Institut 99114.

111 Ebenda, Bl. 26.

112 Brief des Sekretariats Dimitrov an Aleksandrov vom 12. 2. 1943 mit dem handschriftlichen Vermerk Aleksandrovs vom 18. 2. „zur Realisierung der Beschlüsse ist es unerläßlich, eine Besprechung bei mir einzuberufen", RGASPI 17/125/183, Bl. 1.

113 „Auskunft über die Besprechung bei dem Sekretär des ZK der VKP (b) Genosse A. S. Ščerbakov am 24. 4. 1943“, RGVA/K 1p/9a/8, Bl. 118; vgl. Tjulpanov, Der ideologische Kampf gegen den Faschismus, S. 188.

114 Er wurde nicht, wie Heider vermutet (Gründung des Nationalkomitees, S. 19), Leiter des Instituts 99. 